\title{
INDIZI DI UN INVERNO PRECOCE: \\ IL VOTO PROPORZIONALE \\ TRA EQUILIBRIO E CONTINUITÀ
}

\author{
di Roberto Cartocci
}

\section{Non solo un correttivo}

La riforma elettorale del 1993, pur nella sua architettura bifronte e ambigua ${ }^{1}$, modifica in maniera decisiva il sistema elettorale in senso maggioritario. La quota di seggi assegnata con il sistema proporzionale, pensata per attutire $i$ verdetti, prevedibilmente perentori, della competizione maggioritaria a turno unico, rispetto a quest'ultima assume un peso numericamente subordinato.

In conformità allo spirito e alla lettera della legge sia nel 1994 sia nel 1996 il confronto elettorale è stato centrato sulla competizione maggioritaria, in cui le coalizioni e i candidati nei collegi uninominali hanno giocato il ruolo degli attori principali. Nel 1996, poi, il rilievo della competizione maggioritaria è stato ulteriormente accresciuto in virtù del processo di apprendimento istituzionale degli attori ${ }^{2}$. In particolare, nel 1996 un adeguamento decisivo al formato maggioritario è passato attraverso la contrapposizione di due leader di coalizione, designati come capi del futuro esecutivo. Questa personalizzazione dello scontro, così congeniale alle esigenze di semplificazione e drammatizzazione dei media, ha di conseguenza accentuato il rilievo delle due coalizioni principali, che hanno allargato la loro ombra sugli altri attori (la Lega, i candidati di collegio, ecc.), e soprattutto a danno dei contendenti dell'arena proporzionale.

1 Sulle caratteristiche della legge e le complicazioni prodotte dalla compresenza dell'arena maggioritaria e di quella proporzionale cfr. D'Alimonte e Chiaramonte (1995) e Mannheimer e Sani (1994). Sul processo che ne ha accompagnato la gestazione e la nascita, reso particolarmente contorto dalla difficoltà di trovare un compromesso tra una pluralità di interessi, e di angosce, cfr. Pappalardo (1995) e Pasquino (1995).

2 Sul processo di apprendimento istituzionale dei diversi attori (elettori, partiti, coalizioni) cfr. Parisi (1995) e Corbetta e Parisi (1997).

RIVISTA ITALIANA DI SCIENZA POLITICA / a. XXVI, n. 3, dicembre 1996 
Per quanto ignorata dai riflettori della televisione, la competizione proporzionale non aveva perso di interesse agli occhi dei responsabili dei partiti durante la campagna elettorale. Essa non era solo un sicuro passaporto per Montecitorio per i principali leader (quasi tutti con tre presenze nelle liste circoscrizionali), ma anche il metro con cui misurare la forza che i singoli partiti avrebbero potuto esibire nelle successive competizioni dentro e fuori il parlamento, in una fase della transizione politica che resta tuttora aperta a una pluralità di esiti e in cui il processo di ridefinizione dello spettro partitico è ben lungi dall'essersi assestato.

Sia durante la campagna elettorale, sia nei primi mesi della nuova legislatura sono infatti ben visibili i segni delle frizioni tra le diverse componenti delle due coalizioni, tutt'altro che omogenee sul piano degli orientamenti ideologici nonché degli interessi rappresentati. Entrambe le coalizioni sono poi minacciate dal rincorrersi dei progetti, più o meno confusi, di ricostituzione di una nuova forza di centro - e per la quale si segnala una singolare abbondanza di pretendenti alla leadership ${ }^{3}$. Per di più all'interno dell'Ulivo si aggiunge il contrasto fra il progetto di coloro che puntano ad un superamento dei vecchi partiti dando vita a una nuova grande forza di centro-sinistra (l'Ulivo come nuovo partito) e il progetto del segretario del Pds, impegnato invece a costruire un grande partito socialdemocratico, distinto dalle altre componenti dell'Ulivo anche se ad esse alleato.

Per una pluralità di ragioni, dunque, il voto proporzionale rappresenta qualcosa di più che il complemento e il correttivo di un sistema elettorale prevalentemente maggioritario. Per quanto depotenziato in termini di peso elettorale, esso risulta particolarmente rilevante sul piano politico, vale a dire come bussola capace di orientare tattiche e strategie degli attori politici nel prossimo futuro.

La distinzione fra la dimensione puramente elettorale e quella politica risulta fondamentale nell'attuale congiuntura italiana. Sono sempre più evidenti le difficoltà che incontra la trasformazione completa in senso bipolare del sistema partitico italiano, difficoltà che nel lessico giornalistico viene colta dall'espressione «voglia di proporzionale». Le frizioni tra alleati, la

3 A parte i leader delle forze esistenti, non sono mancati i proponimenti in questo senso di Cossiga, della Pivetti e di Di Pietro. 
progressiva riorganizzazione dei partiti, la capacità di attrazione elettorale della Lega e di Rifondazione comunista, i tentativi di ricostituzione di un partito di centro e - last but not least - il nuovo sistema elettorale regionale ${ }^{4}$ sono tutti motivi che riportano in primo piano gli attori-partiti rispetto agli attori-coalizioni. Se queste ultime sono, giuste le intenzioni dei legislatori, le protagoniste della competizione elettorale e si contendono il controllo dell'esecutivo, è anche vero che, definito il nuovo parlamento e uscita dalle urne una maggioranza di governo, la competizione tra le forze politiche si riapre sulla base dei rapporti di forza sanciti dal voto proporzionale.

È certo una forzatura sostenere che il voto maggioritario vale solo il giorno delle elezioni e che il voto proporzionale vale per tutti i mesi successivi. Tuttavia non si deve dimenticare che, a causa del mancato adeguamento in senso maggioritario dello snodo istituzionale governo-parlamento, l'esecutivo mantiene una condizione di debolezza strutturale, tanto più accentuata quanto più ambiguo è l'esito delle urne: le difficoltà interposte da Rifondazione comunista al governo sulla strada della legge finanziaria per il 1997 costituiscono da un lato la prova dei nodi irrisolti in vista di un rafforzamento dell'esecutivo ma al contempo sono anche l'effetto di un indubbio successo elettorale. A parte la differente collocazione rispetto alla maggioranza di governo, la stessa origine hanno i baldanzosi proclami secessionisti lanciati dalla Lega nell'estate ' 96.

Dunque, ben più che elemento complementare e correttivo, il voto proporzionale rappresenta la sponda elettorale della dinamica tra le forze politiche, dentro e fuori le coalizioni, e assume nella congiuntura odierna un rilievo politico primario in quanto costituisce l'espressione delle identità partitiche nuove e vecchie, al di fuori delle considerazioni strategiche che possono orientare la scelta degli elettori nella competizione maggioritaria, fondata su candidati e coalizioni.

In quanto elemento di continuità con il primo cinquantennio repubblicano, il voto proporzionale consente un confronto più preciso con i risultati elettorali fino al 1992, prezioso per lo studioso alla ricerca di continuità e differenze. Va ricordato tuttavia che le nuove regole del voto proporzionale si discostano dalle vecchie in due punti non secopdari Ladifferenza formal-

4 Sul sistema elettorale per le regioni cfr. D limonte (1995) Di Virgilia (1296). 
mente più evidente è costituita dalla soglia di sbarramento del $4 \%$. Si tratta di una differenza il cui significato (quando non la sua esistenza) sicuramente sfugge alla maggioranza degli eletto$\mathrm{ri}^{5}$. Ben più concreta è l'altra differenza rispetto alle regole vigenti prima del 1994: l'impossibilità di esprimere un voto di preferenza. Com'è noto, questa opportunità concessa all'elettore è stata unanimemente riconosciuta dalla metà degli anni ottanta in poi come uno dei puntelli istituzionali che hanno reso possibile la degenerazione dei partiti ${ }^{6}$. Era solo ovvio, quindi, che le nuove regole elettorali escludessero l'espressione della preferenza da parte dell'elettore ${ }^{7}$.

In definitiva, l'abolizione del voto di preferenza depura il voto proporzionale di quelle motivazioni particolaristiche presenti negli elettori e di quegli elementi di personalizzazione della competizione presenti nel voto proporzionale fino al 1992. In altri termini: il voto proporzionale disciplinato dalle nuove regole rappresenta in misura più fedele il consenso per i diversi partiti, attribuito sulla base di pregiudiziali ideologiche (dall'elettorato d'appartenenza) o di valutazioni congiunturali (dall'elettorato d'opinione).

Per la più precisa comparabilità con quello dei decenni precedenti e il sostanziale affrancamento da particolarità locali, connesse alla rosa delle candidature nei singoli collegi, il voto proporzionale risulta la fonte più adeguata per studiare la geografia elettorale del paese e valutarne i mutamenti rispetto al passato. E così possibile trovare risposte agli interrogativi sulle basi territoriali del consenso per i diversi partiti, sulla sopravvivenza delle vecchie fratture e sulla nascita di nuove. Interrogativi tanto più interessanti in una fase contrassegnata da forti tensioni territoriali, prodotte dalle proiezioni geografiche di formazioni sociali diverse e sempre più divaricate ${ }^{8}$.

5 Ben pochi dei quali sanno che una lista deve ottenere oltre un milione e mezzo di voti per sperare di essere ammessa al riparto proporzionale.

6 Cfr. Ruffilli (1987); sui referendum di Segni cfr. Corbetta e Parisi (1994). Sul voto di scambio Cartocci (1990) e la bibliografia ivi citata. Nel 1992 scoppiò poi lo scandalo del voto di scambio a Napoli, che coinvolse i principali esponenti del pentapartito: De Lorenzo (Pli), Di Donato (Psi), Vito (Dc).

$7 \mathrm{Dal}$ momento che la cattura del voto di preferenza costituiva, soprattutto in alcune aree del Mezzogiono, un potente meccanismo di mobilitazione dell'elettorato, non è escluso che il suo venir meno sia all'origine della forte crescita del non voto registrata alle elezioni del 1994 e del 1996, così come viene documentata in questo numero dal saggio di Caramani.

8 Sui rapporti tra politica e società e sulle caratteristiche delle diverse formazioni sociali rinvio al denso lavoro di Bagnasco (1995). 
Se dunque non mancano motivi di interesse per analizzare il voto proporzionale, appare preferibile - nell'attuale fase di convulsa transizione sia politica sia socio-economica - improntare l'analisi ad un atteggiamento di prudenza e di rispetto, adottando un approccio esplorativo, quanto più aperto e scettico nell'interrogare i dati'. Il lettore tuttavia troverà nelle pagine che seguono un filo conduttore tra le diverse fasi dell'esposizione, costituito dall'individuazione di una pluralità di indizi che convergono nell'indicare i segni di una sorprendente continuità del comportamento elettorale degli italiani dopo il terremoto del 1994. Anche le contenute variazioni rilevate convergono poi verso la riduzione delle particolarità locali che si erano espresse nella prima prova delle nuove regole.

\section{Verso una nazionalizzazione della proposta}

Il primo punto da discutere riguarda le caratteristiche della proposta rispetto a cui gli elettori delle diverse circoscrizioni hanno espresso la loro preferenza. Nel complesso le liste che hanno partecipato alla competizione maggioritaria sono state 37 , di cui 21 presenti in una sola circoscrizione (tab. 1). Rispetto al 1994 la contrazione del numero dei partecipanti è netta, in quanto in quella occasione erano apparse sulla scheda grigia ben 64 simboli, di cui 42 presenti in una sola circoscrizione.

Risulta netta dunque la semplificazione del ventaglio di alternative offerte all'elettore e la riduzione della variabilità da una circoscrizione all'altra. D'altra parte occorre aggiungere che la maggioranza delle liste presenti in una sola circoscrizione sono (erano) liste «civetta», presenti solo per consentire la partecipazione di candidati isolati alla competizione maggioritaria nei collegi uninominali, peraltro senza speranza di vittoria ${ }^{10}$ : il

9 Apertura e scetticismo sono i due cardini dell'analisi esplorativa, contrapposta all'analisi che cerca conferme a precise ipotesi (confirmatory). Cfr. Tukey (1978) e Hartwig (1979).

10 Com'è noto si tratta di una disposizione che riguarda solo l'elezione della Camera dei Deputati. Al Senato è consentita nel maggioritario la partecipazione di candidati indipendenti. Di queste liste espressione di candidature locali, nel 1994 fu eletto solo il candidato della Lega di azione meridionale a Taranto-Monte Granaro. Nel 1996 i casi sono stati due: a Taranto Giancarlo Cito ha mantenuto il seggio già conquistato dal suo movimento e a Mirabella Eclano De Mita ha vinto grazie alla desistenza dell'Ulivo. 
processo di assestamento e assimilazione delle nuove regole e la maggiore strutturazione del mercato elettorale hanno fatto desistere molti potenziali competitori, con una semplificazione della proposta che ha riguardato soprattutto le circoscrizioni meridionali. In quest'area le liste con una sola presenza sono scese da 33 a 11.

Il numero assoluto di liste in competizione rappresenta tuttavia un indicatore assai grezzo della struttura della competizione, in quanto del tutto insensibile all'effettivo successo elettorale. Se, anticipando un dato che riguarda la risposta degli elettori, ci si limita alle liste che hanno ottenuto almeno 50.000 voti è possibile apprezzare meglio l'aspetto più rilevante del passaggio 1994-96, vale a dire la sensibile omogeneizzazione della proposta sul territorio nazionale.

Nel 1994 solo quattro liste (Pds, Psi, Ppi e An) erano presenti in tutte le circoscrizioni: una capacità organizzativa che non poteva sorprendere negli eredi dei principali partiti del primo cinquantennio repubblicano. Forza Italia, che pure risultò la lista più votata, era assente in Puglia. Qualche defezione denunciarono pure Rc, i Verdi, la Rete, Ad, il Patto Segni e la Lista Pannella.

Nel 1996 la proposta appare più uniforme sul territorio nazionale: salgono da quattro a sette le liste presenti in tutte le 26 circoscrizioni. Pds e An confermano la loro ubiquità, Forza Italia, Rifondazione e i Verdi riempiono i vuoti lasciati due anni prima mentre Popolari per Prodi (uniti a Svp, Ad e Ud) e CcdCdu mettono a frutto il patrimonio di risorse organizzative e di radicamento ereditati dalla Dc. La Lista Dini manca in due circoscrizioni (Piemonte-2 e Friuli-Venezia Giulia). La Lista Pannella-Sgarbi denuncia carenze organizzative nella provincia centro-settentrionale, fallendo la presentazione nella bassa lombarda (Lombardia-3), nel Triveneto (escluso Veneto-1), in Umbria e nella provincia laziale (Lazio-2). Il Movimento sociale-Fiamma tricolore si presenta in tutte le circoscrizioni del Centro-Sud ma solo in sette del Centro-Nord.

Vi sono poi i casi particolari della Lega Nord e dei socialisti, beninteso assai diversi tra loro. I socialisti di Intini riescono a presentarsi in 10 circoscrizioni, di cui solo 3 del Centro-Nord (Torino, Liguria e Toscana). La Lega aumenta la sua presenza in due circoscrizioni rispetto al 1994, presentandosi anche in Umbria e Marche. Essa resta il principale elemento di differenziazione territoriale della proposta. Naturalmente questa parzia- 
TAB. 1. Caratteristiche della proposta politica nel 1996 e nel 1994. Distribuzione delle liste secondo il numero di circoscrizioni in cui si sono presentate. Sono riportate nominativamente le liste che banno ottenuto almeno 50.000 voti o che si sono presentate in almeno tre circoscrizioni

\begin{tabular}{|c|c|c|c|c|}
\hline \multirow[b]{2}{*}{ Lista } & \multicolumn{2}{|c|}{1996} & \multicolumn{2}{|c|}{1994} \\
\hline & $\begin{array}{c}\text { Totale } \\
\text { circoscriz. }\end{array}$ & $\begin{array}{c}\text { di cui al } \\
\text { Centro-Nord }\end{array}$ & $\begin{array}{l}\text { Totale } \\
\text { circoscriz. }\end{array}$ & $\begin{array}{c}\text { di cui al } \\
\text { Centro-Nord }\end{array}$ \\
\hline Pds & 26 & 14 & 26 & 14 \\
\hline Alleanza nazionale & 26 & 14 & 26 & 14 \\
\hline Forza Italia & 26 & 14 & 25 & 14 \\
\hline Rifondazione comunista & 26 & 14 & 24 & 14 \\
\hline Federaz. dei Verdi & 26 & 14 & 23 & 13 \\
\hline Pop.-Svp-Pri-Ud-Prodi & 26 & 14 & & \\
\hline Ccd-Cdu & 26 & 14 & & \\
\hline Lista Dini & 24 & 12 & & \\
\hline Lista Pannella-Sgarbi & 20 & 9 & 18 & 9 \\
\hline Mov. sociale-Fiamma tricolore & 19 & 7 & & \\
\hline Lega Nord & 14 & 14 & 12 & 12 \\
\hline Socialisti & 10 & 3 & & \\
\hline Lega di azione meridionale & 1 & 0 & 1 & 0 \\
\hline Unione del Nord-est & 1 & 1 & & \\
\hline Union für Süd Tirol & 1 & 1 & & \\
\hline Mani pulite & 5 & 3 & & \\
\hline Partito umanista & 4 & 3 & & \\
\hline Rinnovamento & 3 & 0 & 2 & 1 \\
\hline Noi siciliani - Fsn & 2 & 0 & & \\
\hline Altre 8 liste & 1 & 1 & & \\
\hline Altre 10 liste & 1 & 0 & & \\
\hline Psi & & & 26 & 14 \\
\hline Ppi & & & 26 & 14 \\
\hline La Rete & & & 21 & 11 \\
\hline Alleanza democratica & & & 20 & 10 \\
\hline Patto Segni & & & 16 & 7 \\
\hline Socialdemocrazia & & & 12 & 3 \\
\hline Lega alpina lumbarda & & & 3 & 3 \\
\hline Programma Italia & & & 2 & 0 \\
\hline Lega auton. veneta & & & 2 & 2 \\
\hline Svp & & & 1 & 1 \\
\hline Altre 5 liste & & & 2 & 0 \\
\hline Altre 8 liste & & & 1 & 1 \\
\hline Altre 32 liste & & & 1 & 0 \\
\hline Totale liste & 37 & & 64 & \\
\hline
\end{tabular}

N.B. Sono classificate nel Centro-Nord le circoscrizioni n. 1-14.

Fonte: Istituto Cattaneo su dati ministeriali. 
lità della presenza della Lega è pleonastica rispetto al suo programma e alle sue parole d'ordine, e rispecchia i nuovi termini con cui si è ridefinita nell'ultimo decennio la questione territoriale nel nostro paese.

Rispetto alla presenza della Lega nell'arena proporzionale, il paese risulta diviso esattamente in due a nord di Roma, all'altezza della linea che unisce la foce della Fiora a quella del Tronto. Si tratta di una frattura territoriale che non è nuova nella geografia elettorale. Essa infatti coincide con il confine meridionale della subcultura comunista e con quello settentrionale della zona in cui fino al 1992 si registrava la maggior diffusione del voto di preferenza e dell'astensionismo ${ }^{11}$. Per la prima volta questo confine coincide anche con una radicale diversità della proposta elettorale.

A parte la presenza «dimezzata» della Lega, il panorama della proposta politica mostra i segni inequivocabili della tendenza all'uniformità dopo gli scompensi registrati all'esordio del 1994: diminuisce il numero delle liste in competizione, soprattutto locali, con un conseguente avvicinamento tra circoscrizioni settentrionali e meridionali; passano da quattro a sette le formazioni presenti in tutto il paese, con altre tre liste presenti comunque nella maggioranza delle circoscrizioni.

\section{La risposta: nel segno dell'equilibrio tra destra e sinistra}

Nella molteplicità dei punti di vista da cui guardare ai risultati del voto proporzionale, conviene partire da un confronto con il voto maggioritario. Se, con le salvaguardie necessarie per operare il confronto tra le due arene, si paragonano gli esiti delle due competizioni è possibile inquadrare il significato del voto proporzionale nell'ambito del verdetto complessivo delle elezioni del 21 aprile.

Come illustra la tabella 2, sull'esito finale in termini di seggi (la Camera dei deputati con maggioranza all'Ulivo e Rifondazione comunista) ha agito in maniera decisiva il meccanismo manipolativo del sistema elettorale. Infatti la somma dei voti di lista andata agli alleati nel Polo (44\%) è superiore a quella otte-

11 Per una tematizzazione di questo confine mi permetto di rinviare a un precedente lavoro (Cartocci 1990), in cui si argomenta che tale linea costituisce una frattura più profonda della tradizionale contrapposizione tra zone rosse e bianche. 


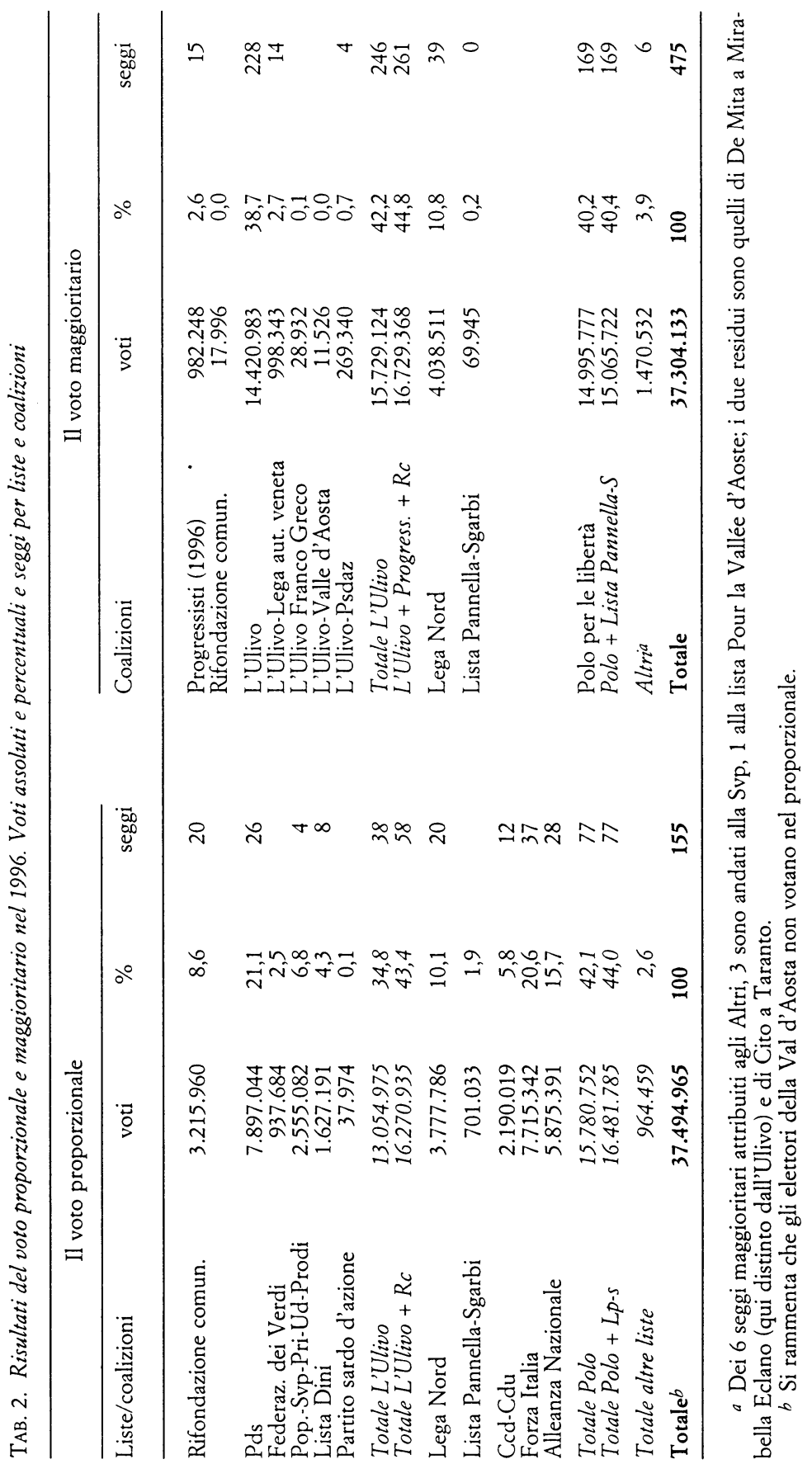


nuta dagli alleati nell'Ulivo (43,3\%, compresa Rc), con uno scarto di oltre 200.000 voti, che si è tradotto in 19 seggi in più ${ }^{12}$. Nell'arena maggioritaria l'Ulivo e i Progressisti hanno ottenuto il 44,8\%, contro il 40,4\% del Polo. Una differenza di oltre un milione e seicentomila voti si è tradotta in 92 seggi in più (oltre a quello di De Mita) che assicurano la maggioranza al centro-sinistra.

Sulle dinamiche competitive nel maggioritario D'Alimonte e Bartolini disegnano un quadro assai dettagliato nel saggio che segue; ad esso rimando anche per un'analisi più sistematica del confronto tra le due arene. In questa sede mi bastano i pochi riferimenti citati per sottolineare come l'arena maggioritaria abbia premiato anche questa volta la coalizione che ha saputo adeguarsi meglio al meccanismo elettorale. Naturalmente anche altri fattori hanno spostato la bilancia in favore dell'Ulivo, come la presenza di una chiara leadership, l'esaurirsi del cosiddetto «nuovismo» e altri ${ }^{13}$; e inoltre - a scanso di equivoci - non è il caso di lamentare le ingiustizie del maggioritario, che ha fatto quello che doveva. Il punto empiricamente rilevante è costituito dal perfetto equilibrio tra le due coalizioni, una volta che esso venga rilevato attraverso il metro proporzionale, sottratto all'appeal dei leader nazionali, alle particolarità dei collegi e alle drastiche sentenze dell'uninominale a turno unico.

Se in un confronto tra contendenti di pari forza la vittoria finale è andata alla coalizione che ha saputo adattarsi meglio alle esigenze del maggioritario, ne consegue che un ruolo particolarmente importante è stato giocato dalla diversa capacità di stringere alleanze, come peraltro Di Virgilio ha messo in rilievo nel suo saggio. In particolare, alla luce degli esiti del voto, sembrano decisivi tre punti: gli accordi di desistenza con Rifondazione e l'arruolamento della Lista Dini come «quarta gamba» del tavolo dell'Ulivo hanno ridotto le defezioni e la dispersione del voto al centro e a sinistra. Sull'altro fronte il Polo ha pagato salata la concorrenza dei nostalgici della Fiamma tricolore ${ }^{14}$, non riuscendo a tradurre in seggi il pur cospicuo numero di voti.

12 Naturalmente i seggi in più sono dovuti non alla differenza di voti ottenuti dagli elettori, ma a quella in termini di cifra elettorale di ciascuna lista, che attraverso lo scorporo penalizza i vincitori nell'arena maggioritaria.

13 Sui risultati del 21 aprile cfr. i saggi di Sani, Fabbrini e Follini su «Il Mulino», n. 3 del 1996.

14 In base ai calcoli di D'Alimonte e Bartolini nel saggio che segue, per la concor- 
$\grave{E}$ proprio da questo accentuato equilibrio tra i due principali schieramenti che il voto proporzionale trae un maggior significato politico ${ }^{15}$. La parità nei rapporti di forza tra i due poli attiva la competizione all'interno degli schieramenti, dato che tutte le singole componenti - anche le minori - possono rivendicare un elevato potere di ricatto. A sinistra è indubbio il successo di Rifondazione rispetto a un Pds rimasto stazionario, anche se diventato il primo partito grazie al sorpasso di Forza Italia. Complessivamente deboli si sono dimostrate le altre gambe del tavolo dell'Ulivo. I popolari, nonostante Prodi e l'alleanza con la Svp, Ad e i laici di Maccanico e La Malfa, non hanno raggiunto il $7 \%$; la Lista Dini ha rischiato di non superare lo sbarramento del $4 \%$ mentre i Verdi ne sono rimasti ampiamente al di sotto.

Sul fronte opposto Forza Italia ha leggermente arretrato ma è riuscita a mantenere a distanza $A n$, che non ha raggiunto il successo sperato (e preconizzato da sondaggi fallaci), anche a causa del buon successo della Fiamma tricolore. Invece gli exdemocristiani del Ccd-Cdu hanno ottenuto un incoraggiante risultato, che rafforza la componente centrista del Polo, ingarbuglia ulteriormente il problema della leadership dell'alleanza e aumenta le tentazioni di dar vita a una nuova Dc. L'incerta collocazione della Lista Pannella-Sgarbi, che solo a una settimana dal voto è entrata ufficialmente nel Polo, ha sicuramente nuociuto alla sua fortuna elettorale, rimasta molto al di sotto del $2 \%{ }^{16}$.

Il chiaro successo della Lega, che ha superato il 10\% dei voti a livello nazionale e il $23 \%$ nelle sei regioni settentrionali, non consiste tanto in queste buone percentuali, quanto nella sua capacità di attirare un numero ancora maggiore di voti nella difficile competizione maggioritaria. Buona parte di questi è andata perduta, dissolta nello scontro con i candidati del Polo e dell'Ulivo, ma ha dimostrato la solidità del consenso leghista o, quanto meno, l'indisponibilità di questi elettori a votare un second best proposto dagli altri schieramenti.

renza nell'uninominale della Fiamma tricolore il Polo ha perso 36 collegi alla Camera e 26 al Senato. Cfr. anche Sani (1996).

15 Sul riverberarsi della competizione proporzionale su quella maggioritaria aveva messo in guardia, ancora prima della prova elettorale, D'Alimonte (1994).

16 A testimonianza delle incertezze che hanno contrassegnato questa lista giova ricordare che Vittorio Sgarbi è stato eletto in Calabria nella lista proporzionale di Forza Italia. 


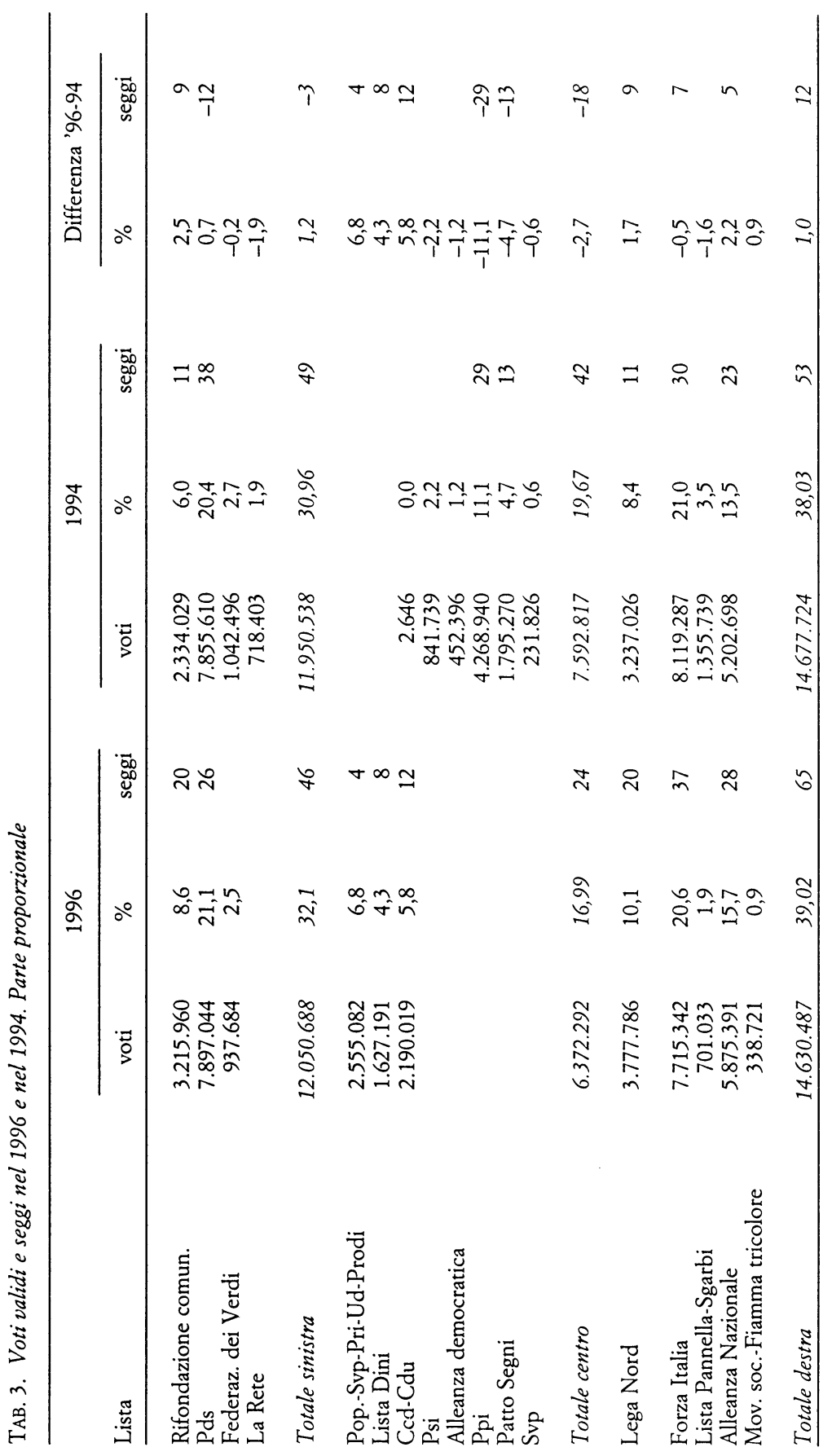




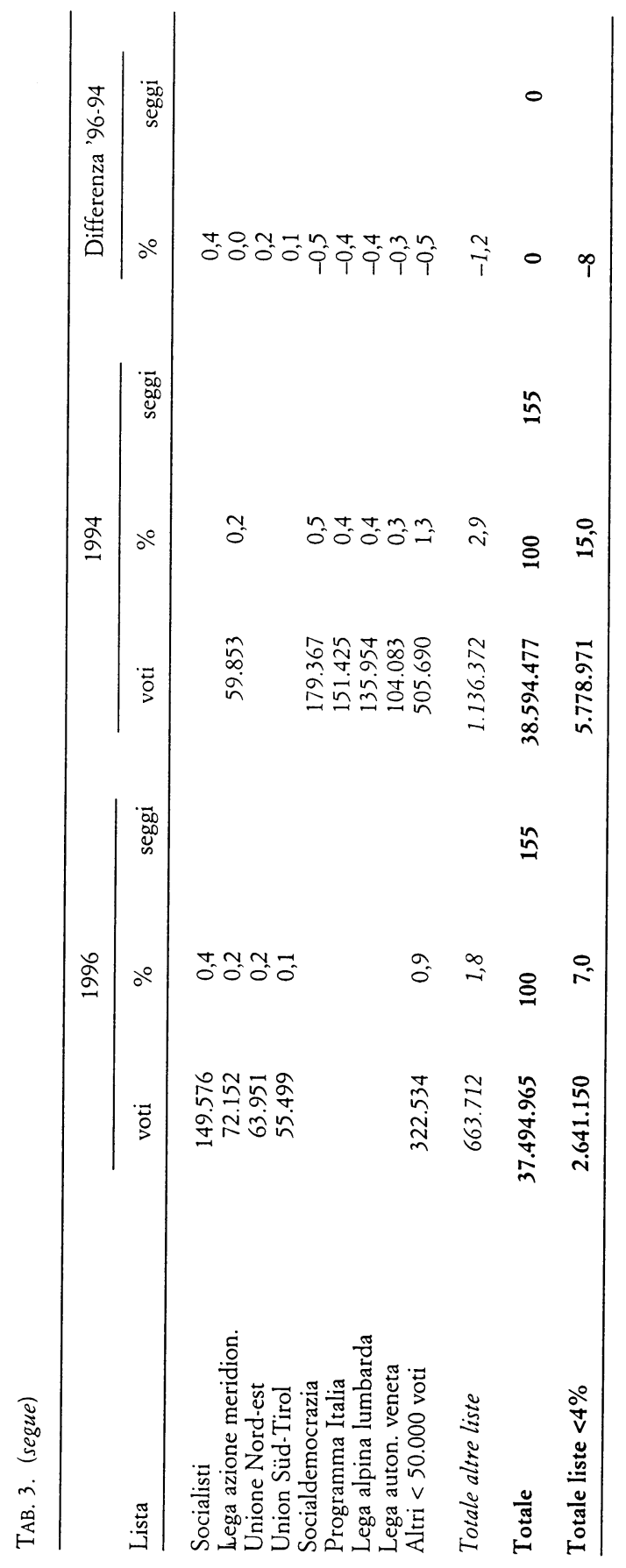


Se si osservano i dati del voto proporzionale attraverso il confronto con il 1994, risulta confermato il processo di apprendimento istituzionale che ha riguardato, in misura diversa, tutti gli attori. In primo luogo gli elettori: nel 1994 erano stati quasi sei milioni coloro che avevano indicato sulla scheda grigia una lista che poi non ha superato la soglia di sbarramento. Il $15 \%$ dei voti era così andato disperso. Due anni dopo la quota di voti andati alle liste minori si è più che dimezzata, fermandosi al $7 \%$ dei voti validi (cfr. tab. 3 ).

Anche la scelta di alcune formazioni indica gli effetti di un processo di apprendimento: il caso più evidente è quello della lista unica che raggruppa con i Popolari per Prodi la Svp, Ad, il Pri e l'Unione democratica di Maccanico. In particolare nel 1994 erano andati dispersi i voti alla Svp, che non poteva da sola ambire a superare il quorum. La scelta di coalizzarsi con il cartello di centro non è stata tuttavia indolore, come vedremo distintamente più avanti. La lezione del ' 94 è stata fatta propria anche da $\mathrm{Ccd}$ e $\mathrm{Cdu}$, la cui decisione di presentarsi uniti è stata premiata dall'elettorato. Analoga scelta è stata compiuta dall'arcipelago di forze, o meglio di debolezze, che ha trovato una casa comune nella Lista Dini (Rinnovamento italiano dello stesso Dini, i Pattisti di Segni e Masi, i socialisti di Boselli e Villetti, il Mid di Sergio Berlinguer), anche se in questo caso l'obiettivo del $4 \%$ è stato raggiunto a fatica.

Con riferimento agli attori-liste, comunque, occorre segnalare che anche nel 1996 si è riproposto un vizio antico della politica italiana: la tendenza delle forze più piccole a difendere gelosamente la loro autonomia anche a costo di essere penalizzate dal sistema elettorale e, con le nuove regole, di penalizzare a loro volta gli alleati. A sinistra i Verdi e a destra i riformatori di Pannella, alla ricerca di visibilità e per riaffermare identità non negoziabili, hanno disperso complessivamente oltre un milione e seicentomila voti.

Diversa considerazione meritano le altre liste minori. La Lega di azione meridionale di Cito doveva esclusivamente «coprire» la candidatura dell'ex sindaco di Taranto, e ha ottenuto peraltro un lusinghiero riscontro nei collegi della provincia, confermandosi un interessante caso di populismo televisivo a scala ridotta. Le altre, spesso esordienti ${ }^{17}$ ed escluse dal gioco

17 Tra le 18 liste non riportate in dettaglio nella tabella 1 né nella tabella 3 vi è anche il Partito sardo d'azione, che ha ottenuto 37.974 voti, come risulta dalla tabella 2 . 
delle alleanze, si può presumere che considerassero già la mera presenza sulla scheda elettorale come un obiettivo in sé, soprattutto nel caso delle liste di disturbo (come Rinnovamento) o di quelle che furbescamente ammiccavano all'elettore esibendo un lessico di moda (Mani pulite, Nord libero autonomia, ecc.). Le principali di queste liste minori, con oltre 50.000 voti, sono state riportate nella tabella 1 e nella tabella 3. L'Unione del NordEst e l'Union Für Süd-Tirol di Eva Klotz hanno incanalato gli orientamenti localisti e regionalisti rispettivamente del Veneto occidentale e dell'Alto Adige. Qui in particolare molti elettori di lingua tedesca non hanno gradito l'apparentamento (con relativa perdita d'identità) della Svp con popolari e laici, premiando la più radicale lista di difesa etnica. I socialisti di Intini, pur presenti in dieci collegi, non hanno raggiunto i 150.000 voti, confermando l'esiguità dei vari rivoli scaturiti dal collasso del Psi.

\section{Frammentazione e continuità}

Per tornare a considerazioni sistemiche, dunque, il quadro dei risultati indica che l'apprendimento delle nuove regole sul versante della risposta degli elettori è stato più accentuato di quello avvenuto sul versante della proposta. Così al calo consistente del numero di liste in competizione ha contribuito un aumento minimo della concentrazione del voto, come indica la tabella 4. L'indice di bipolarismo, vale a dire la somma dei voti dei primi due partiti, è rimasto quasi invariato, dal momento che Pds e Forza Italia si sono scambiati le prime due posizioni con le rispettive frequenze. Il nuovo valore $(41,6)$ resta lontano dal valore assunto nel $1992(45,8)$ e lontanissimo da quello raggiunto nel $1987(60,9)^{18}$. Per apprezzare un aumento (contenuto) della concentrazione conviene seguire D'Alimonte e Bartolini $(1995,434-435)$ che hanno osservato le frequenze cumulate dei primi dodici partiti. Se nel 1994 questi arrivavano a 96,6\%, due anni dopo pervengono al 99,1\%, lasciando fuori meno della centesima parte dei voti validi. Si tratta, come si vede, di un aumento contenuto della concentrazione, riconducibile in buo-

18 Per una serie storica dell'indice di bipolarismo cfr. Corbetta, Parisi e Schadee $(1988,39$ e 406). 
TAB. 4. La concentrazione del voto nel 1994 e nel 1996

\begin{tabular}{cccc}
\hline & \multicolumn{2}{c}{ Percent. cumulate } & \\
\cline { 2 - 3 } n. di liste & 1994 & 1996 & differenza \\
\hline 1 & 21,04 & $21 ;, 06$ & 0,02 \\
2 & 41,39 & 41,64 & 0,25 \\
3 & 54,87 & 57,31 & 2,44 \\
4 & 65,93 & 67,38 & 1,45 \\
5 & 74,32 & 75,96 & 1,64 \\
6 & 80,37 & 82,78 & 2,41 \\
7 & 85,02 & 88,62 & 3,60 \\
8 & 88,53 & 92,96 & 4,42 \\
9 & 91,23 & 95,46 & 4,22 \\
10 & 93,41 & 97,33 & 3,91 \\
11 & 95,28 & 98,23 & 2,95 \\
12 & 96,59 & 99,09 & 2,50 \\
\hline
\end{tabular}

In corsivo le cumulate con i voti delle liste che non hanno ottenuto seggi.

na misura alla crescita del terzo partito (An), e alla maggiore capacità degli attori-elettori di adattarsi ai vincoli del sistema elettorale. Se si limita il confronto con il 1994 ai primi 4-6 partiti, emerge con chiarezza che il biennio non ha ridotto in maniera significativa la dispersione dell'elettorato, come peraltro dimostra il verdetto in termini di seggi: nel 1996 sono state otto le liste che hanno superato il quorum, contro le sette di due anni prima. Nel complesso quindi il sistema partitico si conferma frammentato, con un ulteriore appiattimento rispetto al 1994 in termini di rappresentanza parlamentare.

Appare dunque forte la tentazione di concludere sul punto sottolineando l'accentuazione delle tendenze all'aumento della frammentazione già registrate nelle ultime prove del proporzionale integrale. Questa linea di ragionamento rischia tuttavia di portarci a conclusioni fuorvianti, in quanto contemporaneamente sovrastima e sottostima il grado di frammentazione effettiva. Non si può infatti dimenticare che nel 1994 e nel 1996 rispetto al voto proporzionale è risultata prevalente, come rilievo politico e come peso numerico, la competizione maggioritaria. Il confronto con i dati relativi al vecchio sistema è dunque in buona parte improprio, in quanto mette in parentesi l'esistenza delle coalizioni che hanno radicalmente semplificato la proposta e polarizzato la risposta nell'arena maggioritaria. D'altra parte nella componente proporzionale non sono mancati incentivi che, come abbiamo visto, hanno orientato le decisioni di 
molti attori a confluire in un'unica lista. È presumibile che oltrepassata la soglia di sbarramento - queste molecole instabili tornino a scindersi, aumentando la già elevata entropia del sistema.

Il punto rilevante è, anche in questo caso, non perdere di vista le complicazioni dovute alla compresenza di due diverse arene elettorali, in cui si muove una messe di attori politici distinti in tre livelli - partiti o movimenti, liste presenti sulla scheda grigia, coalizioni presenti sulla scheda rosa. Nella discussione sulla frammentazione non appare tanto rilevante la distribuzione dei voti tra le diverse liste - in un confronto puntuale con i dati di due o quattro anni prima - quanto il significato politico che $\mathrm{i}$ leader sono disposti ad attribuire a questi voti nell'orientare le loro decisioni. Il quadro dei risultati, nonostante la freddezza delle cifre, è oggi intrinsecamente ambiguo. Esso può infatti essere letto dai singoli attori come un incentivo a promuovere fusioni e aggregazioni o alternativamente come un incoraggiamento a valorizzare le singole identità. $\mathrm{Nel}$ quadro uscito dalle urne il 21 aprile mi pare indubbio che prevarrà la seconda alternativa, in modo da far salire il prezzo del futuro ingresso in una delle due coalizioni risultate numericamente così vicine.

Il doppio binario su cui corre la competizione politico-elettorale in questa fase di transizione incentiva le acrobazie tattiche non solo per l'equilibrio tra le coalizioni, ma anche per la sostanziale continuità della risposta degli elettori. Come il lettore avrà osservato, $i$ risultati completi del voto proporzionale nel '94 e nel '96 sono stati raggruppati nella tabella 3 a prescindere dalla composizione delle coalizioni. Con l'esclusione di quelle minori, le liste sono state distinte secondo la collocazione sul continuum sinistra-destra, con un raggruppamento di centro e la Lega, da sola, che sono collocate tra un gruppo di forze di sinistra e uno di destra.

Si tratta cioè di una classificazione che non tiene conto degli allineamenti registrati nella competizione maggioritaria, in quanto discende dal presupposto, più volte enunciato in precedenza, che le due competizioni rispondano a logiche differenti, come differenti risultano gli esiti cui danno luogo. Così Rifondazione comunista è associata a Pds e Verdi (e alla Rete relativamente al 1994), mentre il Movimento sociale-Fiamma tricolore è associato a Fi, An e Lista Pannella-Sgarbi nel raggruppamento di destra. 
Se l'isolamento della Lega Nord esclude dubbi di attribuzione, nell'area di centro la divaricazione rispetto alle alleanze nel maggioritario è radicale. In essa coesistono infatti i diversi tronconi ex-democristiani, sia i popolari per Prodi che - con Svp e Ad - sono confluiti nell'Ulivo, sia il Ccd-Cdu che ha dato vita al Polo. Completa lo schieramento delle forze di centro la Lista Dini, in cui sono confluiti al completo gli stati maggiori del Patto Segni (Masi, Rivera, Fantozzi) e del Psi (Boselli, Villetti, Del Turco).

Se distinguiamo le diverse liste secondo questi quattro raggruppamenti, il confronto tra $\mathrm{i}$ corrispondenti risultati ottenuti nel' 94 e nel '96 fa emergere un quadro di perfetta continuità tra i rapporti di forza tra i diversi segmenti dell'elettorato. Le massime variazioni sono registrate dalla Lega Nord $(+1,7)$ e dalle forze di centro $(-2,7)$. Le formazioni di sinistra guadagnano l' $1,2 \%$; ancora più contenuta è la crescita delle forze di destra $(+1,0)$. L'equilibrio tra perdite e guadagni è infine assicurato dalla contenuta riduzione del voto disperso tra le liste minori ($1,2 \%)^{19}$.

Questo quadro di continuità nelle preferenze degli elettori secondo le grandi aree politiche è confermato dall'analisi più dettagliata, condotta sulle variazioni rilevate per le singole liste. Ho calcolato le variazioni percentuali tra le stesse forze o tra forze solo nominalmente diverse. Per calcolare il cambiamento intervenuto tra il '94 e il ' 96 ho seguito i due criteri enunciati e applicati da Corbetta, Parisi e Schadee nel calcolo dell'indice detto «di instabilità» (1988, 484-493):

a) ho ridotto per quanto possibile l'ampiezza della categoria residuale delle «altre liste», conteggiando le singole variazioni apportate da tutte le liste che in una delle due elezioni hanno ottenuto almeno 50.000 voti; quindi solo le liste che non hanno raggiunto questa soglia confluiscono nella categoria «altri» che compare nella tabella 5 . In tal modo l'ammontare delle variazioni intervenute nell'intervallo '94-'96 non viene artificiosamente depresso dalle compensazioni interne ad una categoria residuale pletorica;

b) ho considerato come elementi unici e comparabili i casi di scissioni, confluenze e alleanze elettorali, per ottemperare ad un'esigenza di segno opposto alla precedente: evitare di gonfia-

19 A livello di grandi aree politiche, l'indice di cambiamento assume il valore di $7,8 / 2=3,9$. 
re artificiosamente l'ammontare delle variazioni registrando come partiti nuovi i meri cambiamenti di sigla o l'esito di divisioni e unificazioni ${ }^{20}$.

In base a questo secondo criterio non pone problemi il confronto tra il voto ad An nel 1994 e la somma dei voti conseguiti da An e dalla Fiamma tricolore nel 1996, essendo quest'ultima nata da una scissione. Il punto più delicato in una fase convulsa come l'attuale riguarda le liste che affollano l'area di centro; e quindi conviene entrare nel dettaglio delle scelte operate per costruire la tabella 5. La scissione avvenuta nel Ppi nella primavera del 1995, precipitata in seguito all'annuncio di Prodi di candidarsi come leader del centro-sinistra, costringe a confrontare i consensi andati al vecchio Ppi nel 1994 con la somma dei voti ottenuti dai Popolari per Prodi con quelli ottenuti da CcdCdu. D'altra parte nel 1996 la lista di Prodi si è presentata insieme ad Ad (Bordon, Ayala, ecc.) e alla Svp. Quindi l'unico confronto possibile è dato dalla somma dei voti andati nel '94 a Ppi, Svp e Ad con la somma dei voti andati due anni dopo ai Popolari per Prodi (uniti a Svp, Ad e Pri) e al Ccd-Cdu. Analoghe considerazioni valgono per l'altra forza di centro, la Lista Dini. In essa sono confluiti, come ho ricordato sopra, i dirigenti nazionali del Patto Segni e del Psi. Dunque la somma dei voti ottenuti da queste due liste nel '94 è messa a confronto con i voti ottenuti nel '96 dalla Lista Dini.

La serie delle corrispondenze e degli addendi che concorrono a costruire l'indice sintetico delle variazioni intervenute tra le due elezioni è riportata nella tabella 5 . Si pone adesso il problema di denominare tale indice. Nella letteratura esso è stato chiamato in una pluralità di modi e calcolato ricorrendo a varie definizioni operative (Masters 1960; Converse 1969; Rae 1971; Dodd 1974) fino alla definitiva sistemazione operata da Przeworski (1975) e soprattutto da Pedersen (1978; 1979; 1983). A quest'ultimo è da far risalire anche l'affermazione del termine volatility, per designare la semisomma dei valori assoluti degli scarti percentuali tra due elezioni successive ${ }^{21}$.

In italiano compare, in tempi pionieristici per la ricerca politologica, la denominazione «indice di variazione», introdotta

20 Lo stesso criterio improntato alla prudenza e alla parsimonia è stato seguito da D'Alimonte e Bartolini (1995, 443).

21 Anche se il termine era stato in precedenza utilizzato nello stesso senso da Asher e Tarrow (1975). 
TAB. 5. Il calcolo dell'indice di cambiamento

\begin{tabular}{|c|c|c|c|c|c|}
\hline Liste o gruppi di liste & 1994 & Liste o gruppi di liste & 1996 & $\begin{array}{l}\text { Differenz } \\
\text { '96-94 }\end{array}$ & $\begin{array}{l}\text { a Valore } \\
\text { assoluto }\end{array}$ \\
\hline Rifondazione comunista & 6 & Rifondazione com. & 8,6 & 2,6 & 2,6 \\
\hline Pds & 20,4 & Pds & 21,1 & 0,7 & 0,7 \\
\hline La Rete & 1,9 & & & $-1,9$ & 1,9 \\
\hline Fed. verdi & 2,7 & Fed. Verdi & 2,5 & $-0,2$ & 0,2 \\
\hline$P p i+S v p+A d$ & 12,9 & Pop. per Prodi + Ccd-Cdu & 12,6 & $-0,3$ & 0,3 \\
\hline Patto Segni + Psi & 6,9 & Lista Dini & 4,3 & $-2,6$ & 2,6 \\
\hline Lega Nord & 8,4 & Lega Nord & 10,1 & 1,7 & 1,7 \\
\hline F. Italia & 21 & F. Italia & 20,6 & $-0,4$ & 0,4 \\
\hline L. Pannella & 3,5 & L. Pannella-Sgarbi & 1,9 & $-1,6$ & 1,6 \\
\hline Alleanza nazionale & 13,5 & An + Mov. soc.-Fiamma tricol. & 16,6 & 3,1 & 3,1 \\
\hline & & Socialisti & 0,4 & 0,4 & 0,4 \\
\hline & & Unione Nord-Est & 0,2 & 0,2 & 0,2 \\
\hline & & Union für Süd-Tirol & 0,1 & 0,1 & 0,1 \\
\hline Lega az. meridionale & 0,2 & Lega az. meridionale & 0,2 & 0 & 0 \\
\hline Socialdemocrazia & 0,5 & & & $-0,5$ & 0,5 \\
\hline Programma Italia & 0,4 & & & $-0,4$ & 0,4 \\
\hline Lega alpina lumbarda & 0,3 & & & $-0,3$ & 0,3 \\
\hline Lega auton. veneta & 0,3 & & & $-0,3$ & 0,3 \\
\hline Altri & 1,1 & & 0,8 & $-0,3$ & 0,3 \\
\hline Totale & 100 & & 100 & 0 & 17,6 \\
\hline \multicolumn{5}{|c|}{ Indice di cambiamento } & 8,8 \\
\hline
\end{tabular}

da Spreafico (1968). Successivamente nelle ricerche più rilevanti troviamo sia il termine «instabilità» (Caciagli e Spreafico 1975, 37; Corbetta, Parisi, Schadee 1988), sia il calco dall'inglese «volatilità» (Bartolini 1986; D’Alimonte e Bartolini 1995).

In effetti entrambe queste denominazioni sono largamente insoddisfacenti in quanto non denotano l'effettivo referente empirico: l'ammontare delle variazioni percentuali intervenute tra due elezioni successive nei voti ottenuti dai singoli partiti. Si tratta dunque di un indice che, giusta la formula matematica, presenta tre caratteristiche: $a$ ) rileva la variazione intercorsa fra il tempo $t_{0}$ e il tempo $t_{1} ; b$ ) si riferisce all'intero sistema partitico; c) sintetizza i risultati aggregati ad un certo livello territoriale, che può essere nazionale (come avviene tipicamente nelle ricerche comparate), ma che può anche essere calcolato per circoscrizione, per collegio, ecc.

Il termine volatilità, oltre ad avere poco corso nel lessico italiano, nel richiamare la proprietà chimico-fisica della tendenza all'evaporazione di un liquido connota una realtà discreta, fatta di atomi e molecole, che suggerisce la propensione al cam- 
biamento di singoli elettori. Esso manca quindi di richiamare la natura sistemica della proprietà rilevata dall'indice ${ }^{22}$. Il termine instabilità coglie meglio questo aspetto sistemico, suggerendo però un'ulteriore connotazione indesiderata: instabilità come caratteristica intrinseca del sistema, o quanto meno riferita ad un processo accertato su un ampio arco di tempo.

Nessuno di questi due termini, in conclusione, rileva e comunica il carattere puntuale/congiunturale, e non processuale/ strutturale, della variazione accertata ${ }^{23}$. La differenza tra le due prospettive temporali, che in termini generali è comunque rilevante, diventa decisiva nell'attuale fase di transizione. In una congiuntura in cui il sistema partitico è in corso di ristrutturazione i singoli passaggi tra due elezioni consecutive assumono un significato particolare, che è opportuno trasmettere con un termine non ambiguo. Inoltre - per lo stesso motivo - le elezioni del '94 e del '96 sono le prime elezioni di una nuova fase della storia politico-elettorale italiana. Sarà dunque legittimo interrogarsi sulla maggiore o minore «instabilità» elettorale del primo cinquantennio repubblicano rispetto alla fase aperta dal passaggio 1992-94.

In altri termini, conviene riservare il termine «instabilità» per denominare una proprietà sistemica e strutturale, visto che - a differenza degli anni scorsi - è possibile ipotizzare una feconda utilizzazione di questa categoria ${ }^{24}$. Mi servirò quindi del termine cambiamento per denominare l'indice costituito dalla semisomma dei valori assoluti delle variazioni percentuali, in

22 Peraltro la tematizzazione della distinzione tra variazioni a livello individuale e a livello aggregato è al centro di una serie di importanti lavori di messa a punto concettuale e terminologica, che hanno distinto ad esempio tra mobilità effettiva e potenziale (riguardo agli individui) e tra le diverse fonti di variazione a livello aggregato, come il ricambio elettorale, ecc. Tra questi studi mi limito a segnalare Barbagli (1979); Parisi (1980); Mannheimer e Sani (1987). Per un recente approfondimento, che fa il punto sulla questione, cfr. Gasperoni (1995).

${ }^{23}$ L'opportunità che con instabilità si dovesse intendere solo una caratteristica strutturale invece che congiunturale era già stata suggerita da Corbetta, Parisi e Schadee $(1988,487)$, che tuttavia avevano poi adottato il termine in considerazione dell'uso prevalso nella letteratura internazionale, distinguendo comunque tra instabilità di lungo e di breve periodo (pp. 29-30).

24 C'è poi un'ulteriore considerazione che sconsiglia l'uso del termine instabilità. Esso infatti viene utilizzato anche in riferimento a una caratteristica del governo. Dal momento che il rafforzamento dell'esecutivo costituisce il principale obiettivo delle riforme istituzionali che si intendono attuare nei prossimi anni, è il caso di circoscrivere e disciplinare il ricorso alla coppia stabilità/instabilità, che presumibilmente sarà assai usata in futuro con riferimento alla robustezza e alla durata degli esecutivi e delle maggioranze parlamentari. 
conformità peraltro con altri lavori relativi alle elezioni '94 e '96 (Vassallo 1997). Certo questa etichetta terminologica non concede nulla alla ricerca di un lessico distinto da quello della vita quotidiana, ma proprio il suo significato nel linguaggio ordinario è quanto di più adeguato allo scopo di suggerire la natura elementare della proprietà rilevata (quanto cambia il risultato di un'elezione rispetto a quella precedente) e la semplicità della sua definizione operativa ${ }^{25}$.

Risolto il problema della denominazione dell'indice e ribadito che la sua definizione operativa è esattamente quella utilizzata dagli altri autori per misurare «l'instabilità» o «la volatilità», resta da commentare il valore assunto dall'indice per il passaggio 1994-96, riportato nella tabella 5. Tale valore è assai basso $(8,8)$ e attesta un inatteso assestamento dei rapporti di forza tra le diverse liste. Si tratta di un messaggio di continuità che contrasta in maniera stridente sia con l'analogo valore assunto dall'indice nel passaggio 1992-94, sia con l'immagine di fluidità che gli osservatori hanno della situazione politica italiana e degli equilibri tra i diversi attori.

La figura 1 illustra questo contrasto stridente. Dopo i decenni di bonaccia elettorale, in cui l'indice di cambiamento dal 1958 al 1983 era rimasto inferiore a 9, negli anni ottanta si era attivato un processo di destrutturazione del sistema partitico, con una crescita del movimento elettorale da parte dei singoli elettori che si era riflesso in un progressivo aumento dell'indice di cambiamento. Le elezioni del 1994 registravano il crollo del vecchio sistema partitico e la nascita del nuovo con un valore altissimo dell'indice ${ }^{26}$; valore che - come attestano D'Alimonte

25 Sufficientemente generico per non evocare una caratteristica strutturale, il termine cambiamento richiederebbe forse l'integrazione dell'attributo «aggregato» per suggerire al lettore la designazione di una proprietà sistemica e non individuale. Tuttavia l'attributo diventa inutile quando si adotti la distinzione tra movimento (la decisione individuale di modificare il proprio voto) e mobilità (intesa come atteggiamento e predisposizione mentale) proposta da Parisi (1980). Il cambiamento, inteso nel senso qui proposto, implica necessariamente il movimento (di molti elettori), che a sua volta implica la mobilità (degli stessi). Non è vero invece il contrario: la disponibilità individuale a cambiare voto non è detto che si traduca in una decisione conseguente; inoltre una somma di movimenti individuali può dar luogo a flussi incrociati che si compensano $\mathrm{e}$ non incidono sull'indice di cambiamento (Gasperoni 1995, 324). Per un approfondimento sulla differenza tra livello individuale e livello aggregato, e per una illustrazione dei rischi di errore inferendo la misura del primo dall'ammontare del secondo, rinvio a Bartolini (1986).

26 La serie storica dell'indice di cambiamento è desunta da Corbetta, Parisi e Schadee (1988) per gli anni 1948-87. Per gli anni più recenti è stata elaborata presso 


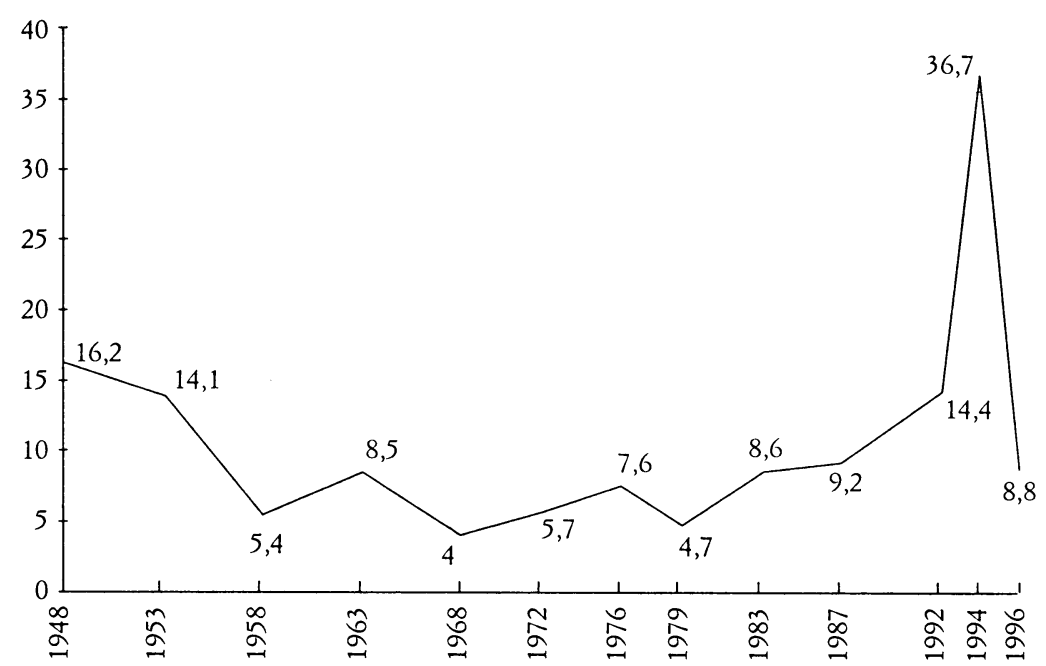

Fonte: Istituto Cattaneo.

FIG. 1. Valori dell'indice di cambiamento 1948-96.

e Bartolini - a livello comparato trova solo quattro precedenti in Europa, tutti riferiti a elezioni di cambiamento di regime ${ }^{27}$. Nel 1996 il valore dell'indice crolla di 28 punti per tornare a un livello inferiore a quello registrato nel passaggio 1983-87.

Il confronto tra le due elezioni tenute col nuovo sistema elettorale suggerisce l'immagine di un repentino congelamento, che solidifica una materia contrassegnata due anni prima da grande effervescenza e fluidità. Questa drastica stabilizzazione della distribuzione dei consensi si contrappone anche al ben più graduale assestamento e assorbimento delle elezioni del 1948. Nel 1953 infatti, cinque anni dopo il 18 aprile, l'indice di cambiamento fu solo di poco inferiore al valore registrato nelle

l'Istituto Cattaneo seguendo i medesimi criteri enunciati nel testo (Vassallo 1997). Le leggere difformità rispetto ai valori pubblicati da D'Alimonte e Bartolini (1995) sono dovute alla diversità di criteri seguiti nell'individuare le corrispondenze tra liste diverse presenti in due elezioni successive. Le variazioni restano comunque contenute e sostanzialmente irrilevanti quanto alle conclusioni che si possono trarre. Ad esempio l'indice relativo al picco del 1994 risulta pari a 37,2, solo mezzo punto in più rispetto al nostro calcolo.

27 Si tratta delle elezioni tedesche del 1919, francesi del 1945, greche del 1950 e 1951 (D'Alimonte e Bartolini 1995, 444). 
elezioni precedenti, segnalando il deflusso dalla Dc dell'elettorato di destra ${ }^{28}$.

Forse l'intervallo tra le due ultime consultazioni è troppo ridotto per consentire il maturare di un quadro di risultati molto diverso; tuttavia le scarse variazioni registrate dall'indice di cambiamento - il cui valore è pari a un quarto di quelle registrate due anni prima - segnalano una sorta di cristallizzazione della forza relativa delle diverse liste. All'equilibrio tra destra e sinistra, accertato in precedenza, si aggiunge il tratto della continuità rispetto a due anni fa. Ne esce così confermata la minore consistenza delle forze di sinistra rispetto a quelle di destra e di conseguenza l'importanza decisiva di due elementi nella vittoria dell'Ulivo: l'aggregazione di due terzi del centro e l'accordo di desistenza ai fini della competizione maggioritaria.

\section{Il cambiamento elettorale nelle circoscrizioni}

In precedenza ho insistito sulla denominazione dell'indice che rileva le variazioni tra un'elezione e l'altra per sottolineare la sua caratteristica di misura congiunturale e al contempo aggregata. Quindi tale assenza di cambiamento non significa di per sé inizio di una nuova stagione di stabilità, la cui esistenza potrà essere accertata solo dalle prossime prove elettorali ${ }^{29}$. In secondo luogo il valore assunto dall'indice di cambiamento non autorizza a proiettare sugli elettori l'immagine di continuità nelle preferenze politiche espresse. Com'è noto, l'indice di cambiamento ci permette deboli inferenze sul comportamento dei singoli. Nel caso-limite di assenza di ricambio nel corpo elettorale, l'indice di cambiamento corrisponde al numero minimo di elettori che hanno modificato la loro scelta. Essi però sono più numerosi dell' $8,8 \%$ registrato nel ' 96 , non solo perché vi è stato un ricambio del corpo elettorale ${ }^{30}$, ma soprattutto poiché si

28 Gli addendi più elevati che concorsero al valore finale dell'indice nel passaggio '48-'53 erano relativi alle perdite della Dc $(-8,4 \%)$ e ai guadagni della destra monarchica e missina $(+4,1$ e $+4,7$ rispettivamente).

29 Come si vede, la necessaria attenzione a differenziare gli elementi congiunturali da quelli strutturali consiglia di non ricorrere al termine instabilità, che evoca una proprietà di tipo strutturale del tutto impropria in questo caso.

30 La riduzione del numero dei voti validi dal 1994 al 1996, documentata nella tabella 3, farebbe pensare a un ricambio contenuto; occorre tuttavia tener conto della vasta e crescente area del non voto, rispetto a cui molti elettori entrano ed escono. 
sono attivati flussi incrociati che l'indice aggregato non riesce a rilevare. Sull'ammontare di questa frazione di elettori che si muovono da un'elezione all'altra possono illuminarci solo gli studi dei flussi, appunto, e i sondaggi. Se si lavora con dati ecologici, l'unica via per stimare più da vicino l'ampiezza del movimento individuale è scendere a livelli di aggregazione inferiori.

Tra i livelli possibili ${ }^{31}$ ho scelto quello circoscrizionale, sia per esigenze di sintesi, sia perché è il livello più elevato che prevede al suo interno una perfetta omogeneità della proposta elettorale riguardo alla parte proporzionale. Detto altrimenti: all'interno di una circoscrizione la scheda grigia è la stessa per tutti i collegi; le risposte degli elettori sono quindi perfettamente comparabili. Invece le differenze tra una circoscrizione e l'altra sono sensibili e lo erano ancora di più nel ' 94 , come abbiamo visto discutendo l'articolazione della proposta. Il calcolo dell'indice di cambiamento per ognuna delle circoscrizioni consente quindi di apprezzare la varietà e la consistenza delle variazioni nelle ventisei singole arene proporzionali, che poi finiscono per fondersi e confondersi nel quadro nazionale presentato nella tabella 5 .

La tabella 6 riporta l'ammontare delle variazioni registrate in ciascuna circoscrizione tra $\mathrm{i}$ diversi addendi che contribuiscono al calcolo dell'indice di cambiamento, così come è stato presentato nella tabella 5 . Le prime due righe della tabella ricordano al lettore le corrispondenze tra le liste presenti nel '96 e quelle presenti nel '94. Per esigenze di spazio, tra le liste minori è evidenziata separatamente solo la Lega di azione meridionale - l'unica che ha ottenuto più di 50.000 voti nelle due elezioni. Per evitare di annegare in un «altri» indistinto la pluralità di liste che hanno arricchito e differenziato la proposta nelle singole circoscrizioni, ho considerato separatamente l'incidenza delle altre liste presenti nel ' 94 e di quelle presenti nel '96. In tal modo è possibile accertare il peso differenziale assunto da queste formazioni minori nelle due tornate elettorali e valutare più puntualmente l'esistenza di un processo di semplificazione (nel caso di riduzione del consenso a queste liste) op-

31 Alla luce della disponibilità immediata di dati, i livelli possibili sono quattro: le sezioni elettorali, i collegi, le circoscrizioni e le grandi ripartizioni costruite fondendo più circoscrizioni. Ovviamente è possibile individuare anche altre ripartizioni riclassificando i dati di livello inferiore, ad esempio utilizzando dati riorganizzati su base provinciale. 


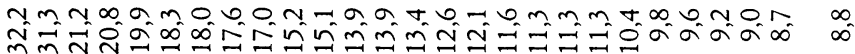

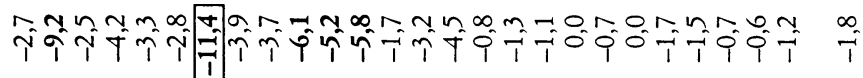

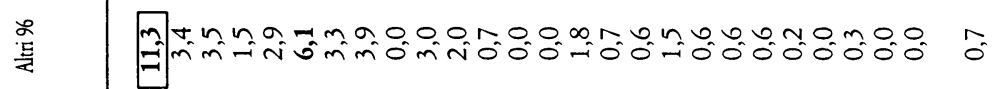

㟧它

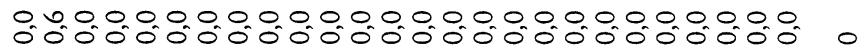

可

芩

जिक्ष̃m

$\stackrel{+}{5}$

空 密

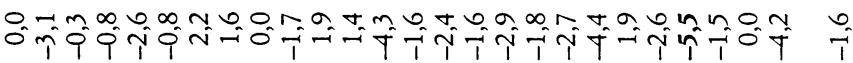

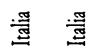

T|⿹𠃌丨

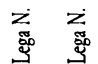

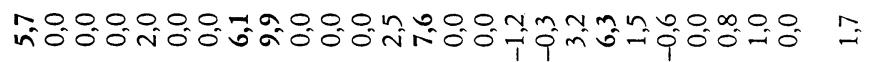

현 쿵 꾼

安实

定+ 吉

Aron $n \pi m n \infty \sigma_{2} n \sigma n+t 0 \infty n=m \infty n m \infty n$

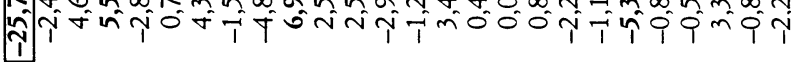

?

:해

क

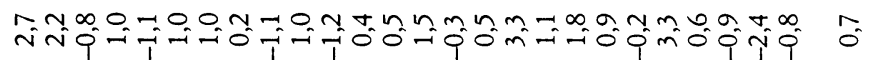

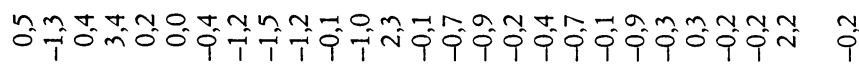

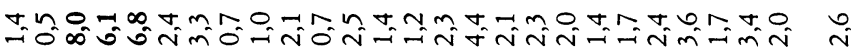

พิ

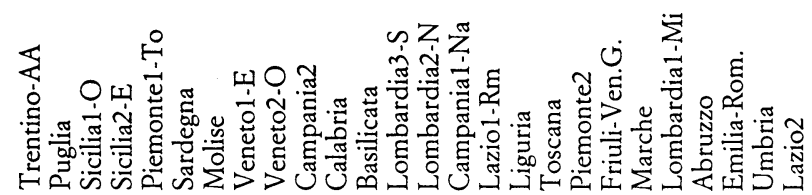


pure, all'opposto, di accentuazione delle particolarità locali ${ }^{32}$. L'ultima colonna riporta il valore dell'indice di cambiamento. L'ultima riga riporta le variazioni accertate tra $\mathrm{i}$ vari addendi a livello nazionale, già presentate nella tabella $5^{33}$. Per agevolare il lettore nel riscontro delle considerazioni che seguono a commento della tabella, ho ordinato le circoscrizioni secondo valori decrescenti dell'indice. Nelle singole caselle, poi, ho evidenziato gli addendi superiori a 10 (in valore assoluto) con un riquadro e quelli superiori a 5 con il grassetto.

Come mostrano i valori dell'indice, in tutte le circoscrizioni, meno la provincia laziale, il cambiamento è stato più elevato di quello registrato a livello nazionale. In complesso la deviazione è inferiore a un punto in cinque circoscrizioni, poste nelle ultime righe della tabella. Un valore più che doppio dell'indice rispetto al dato nazionale è invece registrato in altre sei circoscrizioni. Con un indice superiore al triplo di quello nazionale ci sono poi il Trentino-Alto Adige e la Puglia, che figurano nelle prime due righe. Dunque, come ci si aspettava, l'indice di cambiamento a livello nazionale sottostima fortemente l'ammontare dei movimenti individuali avvenuti il 21 aprile. L'analisi circoscrizionale, se non può darci una misura esatta di questo movimento, ci consente però di correggere in almeno il 14,8\% la frazione dell'elettorato che si è mossa da una lista all'altra ${ }^{34}$, e soprattutto ci offre forti indizi sulla presenza differenziale di questi elettori mobili nelle diverse aree del paese.

Il cambiamento più elevato nel passaggio 1994-96 è avvenuto in Trentino-Alto Adige e Puglia. In questi due casi il valore dell'indice è superiore di oltre 10 punti rispetto a quello della terza circoscrizione e segnala quindi la presenza di peculiarità locali che è opportuno esaminare con cura.

32 Se avessi usato un'unica colonna con i saldi, un valore vicino a 0 avrebbe potuto nascondere sia l'assenza di liste minori nelle due elezioni (e quindi la nazionalizzazione del formato della competizione), sia una presenza consistente e stabile di queste liste (e quindi la persistenza di una particolarità locale della competizione).

33 Per esigenze di coerenza algebrica nella presentazione della tabella, il dato nazionale riferito agli altri è stato scomposto nelle due colonne secondo il seguente criterio: lo scarto di +0,7 registrato nella colonna relativa al 1996 è costituito dal voto andato a Socialisti, Unione del Nord-Est e Union für Süd-Tirol. Lo scarto di $-1,8$ registrato nella colonna relativa al 1994 è costituito dal totale degli altri $(-2,6)$ diminuito della consistenza delle residue altre liste del ' $96(+0,8)$. In tal modo è stato assicurato il pareggio tra scarti positivi e negativi riportati nell'intera riga pur in presenza di due colonne distinte intestate alle altre liste.

34 Si tratta della media dei valori dell'indice di cambiamento circoscrizionale ponderati per i voti validi. 
L'anomalia del Trentino-Alto Adige è in buona parte da far risalire alla grave perdita subita dalla lista Popolari per ProdiSvp-Ud rispetto alla somma dei voti che queste liste, separatamente, avevano conseguito nel 1994. Il confronto tra maggioritario e proporzionale indica che nella competizione proporzionale vi è stata la defezione di molti elettori della Svp ${ }^{35}$, i quali evidentemente hanno considerato un'inaccettabile perdita di identità l'alleanza elettorale con i pur contigui popolari, che in termini di seggi non avrebbe portato alcun vantaggio al partito di difesa etnica. La lista più radicale di $\mathrm{Eva} \mathrm{Klotz}^{36}$ ha raccolto questa domanda insoddisfatta di identità, come attesta l'alto valore dell'addendo «altri 1996». I successi conseguiti dalla Lista Dini e dalla Lega Nord, e la consistenza delle conseguenti variazioni positive, hanno elevato ulteriormente il valore dell'indice.

Nel caso della Puglia l'elevato indice di cambiamento deriva dall'eliminazione dell'anomalia nella proposta del '94. Come si ricorderà, in quella tornata Forza Italia non era presente nel proporzionale. I consistenti suffragi ottenuti nel ' 96 , che ne fanno il primo partito della regione, figurano quindi come la fonte di variazione più consistente, che ha prodotto una corrispondente emorragia da tutte le altre formazioni di centro e di destra, a cominciare da An e dalle altre liste (tra cui figurava un astuto e assonante Programma Italia).

Per quanta prudenza si debba usare nell'inferire i comportamenti individuali dai dati aggregati, la misura del cambiamento registrato in queste due regioni indica nei trentini, negli altoatesini e nei pugliesi gli elettori più mobili del paese. Il significato del movimento (a livello individuale) e del cambiamento (a livello aggregato) è tuttavia ben diverso nei due casi. Nel caso della Puglia le variazioni sono dovute all'eliminazione delle peculiarità locali della proposta, riconducibili ai vizi procedurali riscontrati all'atto della presentazione della lista. Si trattava dunque di un'anomalia di carattere burocratico e transitorio,

35 Nei tre collegi di Appiano, Merano e Bressanone, che comprendono la maggior parte delle vallate di lingua tedesca, i candidati della Svp al maggioritario hanno vinto rispettivamente con il $73 \%, 66 \%$ e $75 \%$. Negli stessi collegi, al proporzionale, la lista comprendente la Svp ha conseguito invece il $38 \%$, il $31 \%$ e il $35 \%$. In questi tre collegi l'Union für Süd-Tirol ha ottenuto una forte affermazione, raccogliendo dal $22 \%$ al $28 \%$ dei voti validi.

36 Com'è noto, la promotrice del movimento è la figlia di Georg Klotz, detto il «martellatore della Val Passiria» e condannato per gli attentati ai tralicci dei primi anni sessanta. 
sanata nella successiva tornata elettorale. Proprio questo carattere in certo senso artificioso della particolarità della proposta del '94 fa della Puglia una sorta di caso paradigmatico di cambiamento elettorale come eliminazione delle anomalie locali e adeguamento ad un pattern nazionale.

Nel caso del Trentino-Alto Adige il cambiamento è dovuto, in misura prevalente, a una causa opposta: la valorizzazione delle particolarità locali, messe in discussione dall'alleanza elettorale della Svp con i centristi dell'Ulivo. Questa circoscrizione appare quindi il caso paradigmatico di cambiamento elettorale come tendenza ad accentuare la diversità, a rivendicare un'identità separata. Se il caso della Puglia indica un cambiamento centripeto, che smussa le differenze, il Trentino-Alto Adige indica un cambiamento centrifugo, che accentua le differenze ${ }^{37}$. Se il primo va nel senso dell'omologazione, il secondo valorizza le particolarità locali.

Si può utilizzare la divaricazione esibita in modo così netto $\mathrm{da}$ queste due regioni per leggere le righe successive della tabella. Le cinque circoscrizioni con un indice superiore a 18 (nell'ordine le due siciliane, la provincia di Torino, la Sardegna e il Molise) sono casi in cui l'elevata variazione registrata dal '94 al '96 è riconducibile a un processo di riduzione delle particolarità locali. In questo senso è infatti interpretabile il dato relativo alle due circoscrizioni siciliane, $i$ cui addendi più elevati sono dovuti alla mancata presentazione della Rete, che aveva ottenuto oltre il $10 \%$ dei voti nel ' 94 , e dal cui bacino evidentemente provengono i voti che hanno fatto lievitare il consenso a $\operatorname{Rc}(+8,0$ e $+6,1)$.

In scala più ridotta un esempio di cambiamento centripeto è costituito dal Molise, in cui la variazione più consistente è dovuta alla radicale contrazione del consenso andato alle liste minori che nel '94 avevano speculato sulla scomparsa della Democrazia cristiana (il Movimento cattolico democratico aveva ottenuto il $4,1 \%$, il Partito popolare progressista il $2,2 \%)^{38}$. Anche

37 Anche nel caso degli aggettivi centripeto e centrifugo è necessaria una puntualizzazione terminologica. In questo caso essi sono utilizzati in senso del tutto diverso da quello applicato nell'analisi sartoriana della meccanica dei sistemi partitici (Sartori 1976; 1982). L'evidente differenza dei contesti mi pare motivo sufficiente per escludere confusioni.

38 Inoltre alla Socialdemocrazia era andato il 4,6\%. Beninteso il Ccd, che era presente in Molise al proporzionale con una lista autonoma, non è stato considerato tra gli altri; i suoi pochi voti $(1,3 \%)$ sono stati sommati a quelli del Ppi per operare il confronto con il '96. 
in Molise, peraltro, il travaso di voti dalla Rete a Rc ha contribuito ad accrescere il valore dell'indice nel senso dell'omogeneizzazione al quadro nazionale (addendi pari rispettivamente a $-2,2$ e a $+3,3)$.

Analoga considerazione anche per la circoscrizione metropolitana di Torino (Piemonte-1), in cui la Rete era risultata particolarmente forte nel '94, potendo contare su un leader di prestigio come Diego Novelli. La mancata presentazione della Rete ha contribuito all'elevato saldo positivo di $\operatorname{Rc}(+6,8)$, il cui valore assoluto pareggia le forti perdite subite da Forza Italia $(-6,7)$, andate a vantaggio di An $(+3,8)$. In sostanza il cambiamento elettorale nella circoscrizione torinese si caratterizza come un'omologazione al quadro nazionale, con un'accentuazione della crescita delle estreme.

In Sardegna nel '94 la particolarità locale era costituita dal successo del Patto Segni (17,9\%), dovuto evidentemente al capitale di popolarità di cui gode nell'isola il leader del movimento referendario. Dopo il ritiro di Segni solo una minima parte di questo elettorato è confluita nella Lista Dini, segnando una variazione di $-14 \%$. Inoltre forti sono stati gli incrementi di An $(+6,9)$ e delle altre liste $(+6,1)$. Quest'ultimo dato può essere solo in parte considerato come un segno di dispersione del voto, in quanto in tale quota confluisce anche il voto al Psdaz, forza tradizionale dell'autonomismo sardo, conteggiato come lista nuova in quanto non presente nel ' 94 .

Le due circoscrizioni venete devono la loro collocazione relativamente alta sulla tabella al forte rafforzamento della Lega $(+6,1$ e $+9,9)$ e alle gravi perdite di Forza Italia $(-7 \text { e }-5,9)^{39}$. Si tratta di un pattern che contraddistingue, anche se con scarti minori, altre quattro circoscrizioni settentrionali: il Friuli-Venezia Giulia, la Lombardia (esclusa Milano) e la provincia piemontese. In questo caso è difficile decidere se queste variazioni sono da intendersi di tipo centripeto o centrifugo. Prevale la prima interpretazione se si considera la Lega un attore stabile del sistema partitico nazionale, anche se con caratteristiche antisistema. In tal caso si mette in rilievo l'azione unificante che la crescita della Lega produce su quella che oggi si chiama Padania, che assume un carattere di unitarietà proprio dalla crescita del partito

39 In Veneto a questo apparente travaso da Fi alla Lega si aggiunge la crescita di An $(+5,2)$ nella circoscrizione Veneto-1 e la contrazione del centro ex-democristiano $(-4,8)$ in Veneto-2. 
di Bossi, più che dalle dimensioni totali del suo elettorato. Prevale piuttosto l'interpretazione centrifuga se si prendono sul serio i propositi eversivi e secessionisti della Lega, che hanno ripreso lena proprio dopo l'affermazione elettorale del Carroccio. In questo caso la forte crescita registrata in queste sei circoscrizioni non può che costituire un motivo di preoccupazione, in quanto è il segnale di un'omogeneizzazione di una vasta area sulla base di un progetto di contrapposizione al centro.

Del tutto diverso il quadro delle variazioni registrate nelle tre circoscrizioni meridionali di Campania-2, Calabria e Basilicata. Il loro indice di cambiamento, che varia tra 15,2 e 13,9, è dovuto principalmente alla contrazione delle «altre liste» e all'incapacità della Lista Dini di raccogliere l'elettorato che nel '94 aveva votato per Patto Segni e Psi. Diversi sono però i beneficiari di queste perdite: An $(+8,0)$ in Calabria, ex-democristiani $(+6,9)$ nella provincia campana e Forza Italia $(+6,5)$ in Basilicata, dove peraltro si aggiungono le variazioni conseguenti alla discreta presenza della Rete due anni fa $(-3,5$ con $+2,5$ per $\mathrm{Rc}$ ). Anche in questi casi, complessivamente, il cambiamento è $\mathrm{da}$ interpretare come erosione di particolarità locali e rafforzamento di alcune delle maggiori forze nazionali, soprattutto di centro e di destra.

Per le circoscrizioni della parte inferiore della tabella, contrassegnate da valori più bassi dell'indice di cambiamento, è più difficile decifrare il significato di variazioni più contenute rispetto ai casi esaminati sopra. Questo gruppo è costituito, è opportuno sottolinearlo, da circoscrizioni metropolitane (Roma, Milano e Napoli), dalle quattro regioni rosse (con l'aggiunta della Liguria), nonché dalla provincia appartata del Lazio e dell'Abruzzo. Da Latina a Ferrara, da Imperia a Pescara, con la significativa aggiunta delle capitali del Nord e del Sud, è un'Italia di mezzo tutt'altro che uniforme sul piano socioeconomico e su quello politico, ma che nel passaggio elettorale '94-'96 è contrassegnata dalla maggiore continuità. Si tratta di un'Italia elettorale che appare più assestata nelle sue scelte - per quanto diverse esse siano - rispetto alle zone a nord del Po o a quelle del Mezzogiorno. E inoltre non mancano altri segnali che confermano la centralità di queste aree rispetto al panorama elettorale del paese, come l'ulteriore ridursi di una già debolissima presenza delle liste minori. Con una scelta elettorale che già nel 1994 aveva premiato solo le liste più solide, due anni dopo queste circoscrizioni esibiscono come variazioni più rilevanti e ri- 
correnti la crescita di An e la contrazione di Fi - con l'eccezione di Napoli, dove i segni sono invertiti.

In conclusione, il quadro delle variazioni rilevate nelle singole circoscrizioni conferma che nel complesso i flussi di elettori mobili sono stati più consistenti di quanto attesti l'indice di cambiamento a livello nazionale. Quest'analisi corregge quindi l'immagine di continuità che il mero confronto tra i dati complessivi (tabb. 3 e 5) suggeriva. Non si tratta tuttavia di una smentita bensì di una precisazione: se si tiene a mente la distinzione tra cambiamento centripeto e cambiamento centrifugo, buona parte delle variazioni accertate (a livello aggregato) e dei movimenti inferiti (a livello individuale) è avvenuta in direzione centripeta, nel senso dell'elisione delle particolarità locali. Ben più ridotta appare l'ampiezza delle variazioni centrifughe - beninteso a condizione di non considerare tali i consistenti flussi di elettori che nel Nord hanno premiato la proposta radicale della Lega. Nell'ipotesi più prudente tale crescita registra un'accentuazione delle differenze tra il Nord e le altre zone del paese, ma con una doverosa precisazione: la Padania leghista appare una regione vasta ma acefala. Le due metropoli di Milano e Torino mostrano un quadro elettorale assai diverso da quello del resto del Nord, più o meno profondo. Peraltro, le circoscrizioni metropolitane di Milano, Roma e Napoli si segnalano per bassi livelli di variazione rispetto al '94. Se il ruolo delle capitali è quello di anticipare i futuri movimenti della periferia, potrebbe essere un indizio che rafforza l'impressione di un rapido congelamento dei rapporti di forza tra le principali forze politiche, in un mercato che in pochi anni si è fatto assai vischioso.

\section{La questione della geografia elettorale}

L'analisi condotta per circoscrizioni ci ha permesso di osservare da vicino la varietà dei flussi che nelle diverse zone si sono incrociati nell'ultimo biennio. L'analisi è stata condotta peraltro presupponendo l'esistenza, e la conoscenza, dei diversi panorami elettorali che coesistono nel nostro paese. La grande varietà della geografia elettorale italiana ha prodotto negli ultimi decenni una molteplicità di studi, che hanno accertato quanto profonde siano le differenze territoriali fin dalle prime prove elettorali, dopo l'Unità. Topos che è stato ripreso da vari ricer- 
catori anche dopo le elezioni del '94, e più in generale in seguito alla nascita della Lega ${ }^{40}$.

In questa vastissima letteratura, un problema particolare riguarda l'interrogativo su quante siano le Italie elettorali. Dopo le elezioni del 1992, alle tradizionali divisioni in quattro (o cinque) zone, derivate dalla classica ricerca condotta dal Cattaneo negli anni sessanta, si è sostituita una divisione in tre grandi zone, che prendeva atto della crisi della presenza della Dc nella sua area di insediamento storico (il Nord-Est) e della progressiva omogeneizzazione di tutto il Nord (la Padania) nel segno dell'espansione leghista (Sani 1992; 1993).

Questa divisione tripartita appare ancor oggi imprescindibile, quantomeno come articolazione preliminare indispensabile ad ogni analisi accurata, dal momento che essa è dettata dalle differenze più macroscopiche in termini di proposta e di risposta degli elettor ${ }^{41}$. Come ho sottolineato in precedenza, sul versante della proposta l'Italia è divisa in due parti dalla presenza/ assenza della Lega, all'altezza della linea che corre da Grosseto ad Ascoli Piceno. Sul versante della risposta l'area a nord di questa linea è a sua volta distinta dal corso del Po tra la zona di maggiore espansione della Lega e le regioni di insediamento della subcultura socialcomunista.

La tabella 7 riporta succintamente la diffusione delle principali forze politiche in queste tre aree nel $1996^{42}$, con alcuni possibili confronti con il 1994. I tratti distintivi delle tre aree escono confermati, con la prevalenza del duopolio Fi-Lega al Nord e del solo Pds nella Zona rossa, e con un sostanziale equilibrio fra le tre forze maggiori al Centro-Sud. Più interessanti sono le variazioni nel biennio che si possono percepire su questa scala territoriale, e che attestano un processo di contrazione delle differenze fra le tre zone. Nel passaggio 1994-96 il Pds presenta le variazioni minori, con una minima perdita nella sua roccaforte e un aumento al Nord e al Sud. An, che nel '94 era più forte nelle regioni meridionali, aumenta dove era più debole, con oltre

40 Cfr. ad esempio i lavori di Diamanti (1995), Natale (1994), Ricolfi (1995; 1996), Agnew (1995). Per le più recenti tematizzazioni del significato da attribuire all'analisi ecologica del voto cfr. Agnew (1996) e Bagnasco (1996).

41 Sull'inderogabilità di queste considerazioni per procedere a distinzioni successive rinvio a una mia precedente puntualizzazione (Cartocci 1996).

42 Il Nord comprende le circoscrizioni 1-10, la Zona rossa le circoscrizioni 11.-14 (Emilia-Romagna, Toscana, Marche e Umbria); tutte le altre circoscrizioni confluiscono nel Sud. Naturalmente la Val d'Aosta è esclusa. 


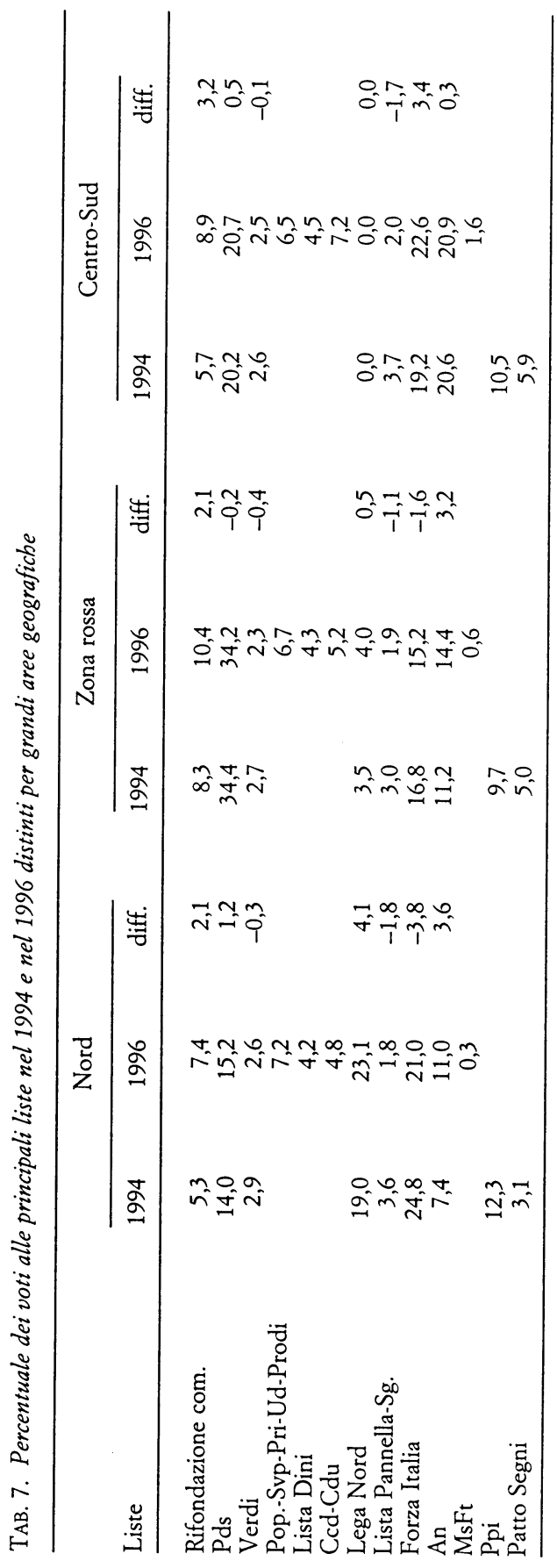


tre punti guadagnati nelle regioni centro-settentrionali. Forza Italia, che tra le forze maggiori era quella distribuita più uniformemente, perde consensi al Nord (dove viene superata dalla Lega) per guadagnarne al Sud (dove diventa il primo partito). Anche Rifondazione si espande in ogni zona, e in particolare nelle regioni centro-meridionali; di converso Verdi e Lista Pannella calano uniformemente. Tra le forze di centro Popolari e Lista Dini sono equamente diffusi, mentre il Ccd-Cdu ha un bacino di consensi più sbilanciato verso il Centro-Sud.

Dunque le tre Italie elettorali escono confermate dall'esito del 21 aprile, ma con un'apprezzabile riduzione delle reciproche differenze in termini di voto ai principali partiti del polo di centro-destra e a Rifondazione comunista. Resta però la complicazione della Lega, che accrescendo i suoi consensi al Nord (ma anche nella Zona rossa) allontana quest'area dal resto del paese. Granitico appare poi l'arroccamento del Pds nelle tradizionali regioni rosse. Abbiamo già osservato (tab. 3 ) come le variazioni di questo partito siano minime a livello nazionale $(+0,7 \%)$. Anche a livello di circoscrizione le variazioni sono molto più esigue di quelle esibite da tutte le altre forze politiche. Tra le forze politiche maggiori, la colonna del Pds nella tabella 6 è quella che contiene le variazioni più ridotte: in quindici casi gli addendi non superano \pm 1 , con un massimo di $+3,3$. La tabella 7 non $\mathrm{fa}$ che sintetizzare questo quadro di radicale rigidità del consenso al partito, che contraddistingue sia il suo tradizionale bacino geografico di raccolta, sia le altre zone del paese.

Sul piano analitico è possibile rispondere al duplice interrogativo su quanto profondamente queste tre zone si distinguano l'una dall'altra e su quanto risultino internamente omogenee: ci soccorre l'analisi della varianza e il calcolo del coefficiente eta ${ }^{2}$. Com'è noto, esso misura la porzione della varianza di una variabile cardinale (come la percentuale a un partito nei collegi) che è riconducibile alle differenze fra le diverse categorie (come le tre zone sopra definite). Quanto più alto (cioè vicino $a+1)$ è il coefficiente, tanto più elevata sarà l'omogeneità interna a ciascuna zona e quindi tanto maggiore sarà la differenza tra una zona e l'altra. L'applicazione dell'analisi della varianza ai dati relativi alle elezioni precedenti ci permette anche di rispondere all'interrogativo sollecitato dalla rilevazione dell'indice di cambiamento a livello di circoscrizioni. Dalla discussione della tabella 6 avevo tratto la conclusione che il pur ridotto cambiamento registrato nel biennio $1994-96$ era in buona misura ri- 
TAB. 8. Matrice dei coefficienti eta ${ }^{2}$ calcolati per la relazione tra le principali liste alle elezioni del 1992, 1994, 1996 e le suddivisioni delle circoscrizioni in 3, 4 e 5 zone

\begin{tabular}{|c|c|c|c|c|}
\hline \multirow[b]{2}{*}{ Liste/anni } & \multirow[b]{2}{*}{$\%$} & \multicolumn{3}{|c|}{ Valori di eta ${ }^{2}$} \\
\hline & & 3 zone & 4 zone & 5 zone \\
\hline Dc 92 & 29,7 & 0,45 & 0,48 & 0,51 \\
\hline Ppi 94 & 11,1 & 0,04 & 0,10 & 0,10 \\
\hline Pop. per Prodi 96 & 6,8 & 0,01 & 0,11 & 0,12 \\
\hline Ccd-Cdu 96 & 5,8 & 0,18 & 0,19 & 0,26 \\
\hline Pds 92 & 16,1 & 0,54 & 0,56 & 0,58 \\
\hline Pds 94 & 20,4 & 0,56 & 0,57 & 0,58 \\
\hline Pds 96 & 21,1 & 0,54 & 0,57 & 0,57 \\
\hline Rc 92 & 5,6 & 0,27 & 0,33 & 0,36 \\
\hline Rc 94 & 6,0 & 0,26 & 0,29 & 0,30 \\
\hline Rc 96 & 8,6 & 0,15 & 0,23 & 0,26 \\
\hline Msi 92 & 5,4 & 0,32 & 0,32 & 0,32 \\
\hline An 94 & 13,5 & 0,59 & 0,59 & 0,60 \\
\hline An 96 & 15,7 & 0,44 & 0,45 & 0,57 \\
\hline Psi 92 & 13,6 & 0,18 & 0,20 & 0,27 \\
\hline $\mathrm{Fi} 94$ & 21,0 & 0,20 & 0,22 & 0,27 \\
\hline Fi 96 & 20,6 & 0,17 & 0,22 & 0,36 \\
\hline Lega $92^{a}$ & 8,7 & 0,51 & 0,55 & 0,55 \\
\hline Lega 94a & 8,4 & 0,48 & 0,48 & 0,48 \\
\hline Lega $96^{a}$ & 10,1 & 0,47 & 0,48 & 0,48 \\
\hline \multicolumn{5}{|c|}{ medie ponderate annuali } \\
\hline media 92 & & 0,41 & 0,43 & 0,46 \\
\hline media 94 & & 0,37 & 0,39 & 0,40 \\
\hline media 96 & & 0,33 & 0,36 & 0,43 \\
\hline
\end{tabular}
li (n. 1-14).

conducibile a una direzione centripeta, di nazionalizzazione del voto. Si tratta quindi di osservare se e quanto le diverse Italie elettorali si sono avvicinate.

Nella tabella 8 (seconda colonna) sono riportati i valori di eta $^{2}$ calcolati ponendo come variabili dipendenti le percentuali alle principali liste registrate nei 474 collegi nel 1992, 1994 e 1996, e come variabile indipendente la suddivisione in tre zone secondo i criteri enunciati. Nel caso della Lega, per evitare di enfatizzare in misura inaccettabile dal punto di vista statistico la 
sua presenza in una sola metà del paese, ho escluso dal computo $i$ collegi del Centro-Sud in cui non si è mai presentata ${ }^{43}$.

Dalla tabella risulta che nel 1992 la geografia elettorale italiana era strutturata principalmente intorno alla diffusione del Pds $\left(\right.$ eta $\left.^{2}=0,54\right)$, della Lega $\left(\right.$ eta $\left.^{2}=0,51\right)$ e della Dc $\left(\right.$ eta $^{2}=$ $0,45)$. Dunque circa metà della variabilità accertata nella diffusione di queste liste era da ricondurre alle differenze tra una zona e l'altra. A parte la Lega, erano chiaramente riconoscibili i segni della continuità con la geografia elettorale dei primi anni del dopoguerra, fondata sulla contrapposizione Dc-Pci.

Nel 1994 si accentua leggermente la concentrazione del Pds, mentre la Lega risulta un po' meno arroccata a nord del Po. Fra le forze di destra, la crescita di An differenzia ulteriormente il Centro-Sud dalle altre zone con una fortissima crescita del coefficiente rispetto a quello relativo al Msi nel $1992\left(\right.$ eta $^{2}=0,59$ contro 0,32 ). In altri termini, dopo le elezioni del ' 94 la concentrazione del voto ad An a sud della linea Grosseto-Ascoli disegna una frattura territoriale più profonda della concentrazione del voto pidiessino nella Zona rossa. Invece Forza Italia appare, tra le liste principali, quella diffusa in maniera più uniforme $\left(\right.$ eta $\left.^{2}=0,20\right)$. Il Ppi, nella sua drastica contrazione rispetto al peso elettorale della Dc, perde - oltre che elettori e seggi - anche ogni accentuazione territoriale $\left(\right.$ eta $\left.^{2}=0,04\right)$.

Nel 1996 i valori di eta ${ }^{2}$ diminuiscono leggermente per Pds, Fi e Lega, in maniera più sensibile per Rc. Calano drasticamente per An, grazie ai guadagni ottenuti nei collegi del Nord e della Zona rossa. I residui voti popolari risultano ancora più equidistribuiti di quelli del Ppi nel '94, mentre il Ccd-Cdu, con il suo bacino squilibrato verso il Centro-Sud, esordisce con un valore di eta ${ }^{2}$ superiore a quello di Rc.

Dal punto di vista statistico, dunque, il duplice passaggio elettorale dal '92 al '96 attraverso il cambiamento di molti attori principali ha avvicinato il panorama elettorale delle tre aree del paese, come indicano i valori medi riportati nella tabella in basso, calcolati ponderando i coefficienti delle varie liste sulla base

43 La decisione è cruciale nel determinare l'esito dell'analisi della varianza. Aver escluso come casi mancanti $\mathrm{i}$ collegi in cui la Lega non si è mai presentata significa privilegiare il momento della risposta su quello della proposta. La via alternativa (effettuare l'analisi su tutti i collegi) avrebbe prodotto un coefficiente molto più elevato, dovuto alla presenza dimezzata della Lega, ma insensibile alle variazioni di consensi registrate dove essa era effettivamente presente. 
dell'ampiezza del consenso ottenuto (riportata nella prima colonna di tab. 8).

Sempre sul piano statistico è possibile anche esplorare soluzioni più articolate, suddividendo le dieci circoscrizioni settentrionali in due diverse classi (Nord-Ovest e Triveneto) al fine di esplorare i riflessi elettorali della nuova realtà economica (e politica) disegnata dal peculiare sviluppo delle regioni nordorientali. In generale, aumentando una modalità della variabile indipendente, il coefficiente eta ${ }^{2}$ tende a crescere in quanto aumentano i gradi di libertà, vale a dire le possibilità di distribuire i casi in classi internamente omogenee. Nel nostro caso la separazione dei collegi del Nord-Ovest da quelli del Nord-Est produce effetti molto differenziati tra le diverse forze politiche. Gli incrementi più rilevanti, riportati nella seconda colonna della tabella 8, riguardano i voti dei popolari nel ' 94 e nel ' 96 . Infatti la distinzione del Nord-Est dalle altre regioni settentrionali consente di apprezzare i consensi dovuti alle residue tracce dell'insediamento democristiano nel Triveneto. Anche la distribuzione del voto a Rc e Fi risulta più nitida con la divisione in due parti del Nord, che fa affiorare la loro maggiore diffusione nel triangolo industriale. Pressoché nulli sono gli effetti sui coefficienti relativi al Pds, sui cui voti, legati alle aree più urbanizzate e di più antica industrializzazione, la separazione tra Est e Ovest non costituisce un criterio rilevante.

Può forse suonare sorprendente constatare che quest'ulteriore suddivisione non produca un aumento dei valori di eta ${ }^{2}$ della Lega, considerata nella pubblicistica corrente l'espressione politica delle aree a industrializzazione diffusa concentrate nel Nord-Est. In effetti la distribuzione della Lega risulta ineguale sia nelle regioni del Nord-Ovest, sia nel Triveneto. Nelle prime infatti essa è molto debole nelle aree metropolitane di Torino e Milano e in tutta la Liguria. Nel secondo è debole in TrentinoAlto Adige e lungo la costa da Rovigo a Trieste. In altri termini, per rilevare la presenza differenziale della Lega nelle regioni del Nord questa suddivisione non migliora il potere di risoluzione della classificazione. Occorrerebbe un criterio geograficamente più sofisticato, come isolare la provincia prealpina dalle aree costiere e metropolitane, per ricostruire le caratteristiche già osservate dagli studiosi del fenomeno leghista. Esso tuttavia appare, oggi, intollerabilmente ad boc per mantenere un suo autonomo valore euristico e non farci cadere nella tautologia.

Lungo la stessa linea di ragionamento, risulta più proficua 
la divisione in due parti della vasta area del Centro-Sud. La conseguente suddivisione dell'Italia in cinque aree, ottenuta distinguendo Lazio, Abruzzo, Molise e Sardegna dal profondo Sud continentale e dalla Sicilia, permette vantaggi apprezzabili nella rilevazione della diffusione differenziale di molte forze politiche nel 1996. Come mostra l'ultima colonna a destra nella tabella 8 , i valori di eta ${ }^{2}$ delle diverse liste non variano molto per gli anni '92 e '94 rispetto ai corrispondenti valori calcolati mantenendo in un'unica categoria le dodici circoscrizioni centromeridionali. L'ulteriore distinzione risulta invece opportuna per il voto del 21 aprile. Essa consente di registrare, nell'ambito di questa metà del paese, la diffusione differenziale delle tre liste di centro-destra. Infatti il valore di eta ${ }^{2}$ di Forza Italia passa da 0,22 a 0,36, quello di An da 0,45 a 0,57, e quello degli ex democristiani del Polo da 0,19 a 0,26. Questa distinzione riesce così a tener conto e a segnalare:

a) la maggiore presenza di Fi nell'estremo Sud e in Sicilia rispetto al Centro $(25,3 \%$ contro $17,9 \%$ in termini di percentuali medie di collegio);

b) i più elevati consensi ottenuti da An al Centro rispetto al profondo Sud $(25,1 \%$ contro $18,1 \%)$;

c) la concentrazione del Ccd-Cdu all'estremo Sud $(8,1 \%$ contro il $5,7 \%$ registrato nelle quattro regioni centrali a ridosso della Zona rossa).

Il quadro di risultati esposto nella tabella 8 delinea dunque una risposta inattesa all'interrogativo su quante siano le Italie elettorali uscite dalle urne il 21 aprile. I confini che segnano la divisione in tre parti mostrano segni di erosione (i valori medi di eta ${ }^{2}$ scendono da 0,41 a 0,33 ). D'altra parte la distinzione tra Nord-Ovest e Nord-Est non migliora in maniera significativa il potere di risoluzione della classificazione (i valori medi di eta ${ }^{2}$ salgono di 2-3 punti) e al contempo conferma la tendenza all'erosione delle differenze dal ' 92 in poi.

Risulta invece rilevante la distinzione tra le quattro regioni centrali (Lazio, Abruzzo, Molise e Sardegna) e il resto del Sud, soprattutto per quanto riguarda la diffusione dell'elettorato di centro-destra. Infatti questa distinzione non solo consente di pervenire a valori sensibilmente più elevati di eta ${ }^{2}$ per il 1996, ma appare anche una divisione più profonda che nel 1994. Quindi, in altri termini, se non mancano segnali di erosione delle differenze nella metà centro-settentrionale del paese, contemporaneamente si manifestano indizi di una radicalizzazione 
delle differenze nell'altra metà, in cui si è accentuata la competizione a destra fra An e Forza Italia ${ }^{44}$. D'altra parte l'interesse per questa divisione della metà centro-meridionale del paese diventa ancora più evidente se si allarga il campo oltre $i$ voti di lista. Ad esempio, considerando l'ammontare dei voti non validi la separazione delle aree di centro dal profondo Sud risulterebbe ancora più raccomandabile in vista dell'individuazione di aree sufficientemente differenziate dal punto di vista del comportamento elettorale (e non solo del comportamento di voto).

\section{Rilievi conclusivi}

In sede di bilancio occorre tornare a sottolineare i molteplici segnali di assestamento della risposta degli elettori nell'alveo scavato nel 1994. Ridotto sul versante della proposta il ventaglio delle liste in competizione, gli elettori hanno dimostrato di aver saputo adeguarsi alle nuove regole riducendo anche il capitale di voti in precedenza dissipato tra le formazioni minori. Ma le gerarchie tra le prime sei-sette liste non si sono modificate e quindi non è emersa un'apprezzabile semplificazione del sistema partitico. Questa persistente frammentazione, congiunta all'equilibrio tra sinistra e destra, ha finito per aumentare il rilievo politico della competizione proporzionale. Nato come ammortizzatore e correttivo rispetto al drastico meccanismo di trasformazione dei voti in seggi dell'uninominale a turno unico, il proporzionale esce dalle urne del 21 aprile come la bussola per orientare la competizione tra le diverse liste, sia all'interno delle rispettive coalizioni, sia nella prospettiva di una loro ridefinizione: ad esempio certificando il potenziale di coalizione e di ricatto di liste pur esigue e aumentando le tentazioni di ricostituire una forza di centro.

Il confronto con il 1994 indica che in generale sono stati pochi gli elettori che hanno cambiato il loro orientamento di voto $^{45}$. I dati complessivi attestano un cambiamento così ridotto

$44 \mathrm{Nel} 1994$, nei 181 collegi delle dodici circoscrizioni centro-meridionali in cui erano presenti entrambe le forze, il coefficiente di correlazione tra il voto ad An e quello a Fi era di -0,36; nel 1996 il coefficiente (su tutti i 215 collegi) sale a -0,48.

45 I dati di un ampio sondaggio post-elettorale promosso dall'Istituto Cattaneo confermano pienamente questa tendenza, secondo cui le elezioni del 21 aprile risultano elezioni di assestamento (Corbetta e Parisi 1997b). 
rispetto al 1994 da suggerire l'immagine di una gelata precoce, che ha irrigidito prima del previsto gli orientamenti del corpo elettorale. L'analisi più dettagliata, condotta al livello delle circoscrizioni, da un lato corregge quest'immagine, attestando che gli elettori mobili sono almeno il doppio di quell' $8,8 \%$ suggerito dall'indice di cambiamento. D'altro lato quest'analisi localizzata conferma che buona parte delle variazioni di voto è da ricondurre a movimenti di assestamento, che eliminano o riducono quelle particolarità locali che, favorite da un sistema elettorale all'esordio, erano emerse numerose nel ' 94 . Dunque il cambiamento, già contenuto in termini complessivi, è avvenuto in buona parte nel senso, che ho definito centripeto, di un rafforzamento delle caratteristiche «nazionali» della competizione.

In questo quadro di assestamento e di stabilizzazione degli attori del sistema partitico, un elemento di particolare rigidità è costituito dal seguito del Pds. A livello nazionale la variazione percentuale riscontrata dal '94 al '96 è inferiore a un punto. D'altronde l'analisi circoscrizionale ci consente di escludere che il dato complessivo nasconda più ampie variazioni in più e in meno nelle diverse aree del paese. Di tutte le principali liste, il Pds è l'unica che confermi a livello delle circoscrizioni la staticità della base elettorale che risulta dal totale nazionale. Si tratta di una rigidità stupefacente, che evoca un'opinione pubblica poco sensibile, che non disapprova ma neppure apprezza quanto il partito nell'ultimo biennio ha fatto per rinnovarsi in termini di leadership, di strategia e di programma. Quest'assenza di elasticità delle basi elettorali del Pds pone al partito un problema di strategia delle alleanze e - a posteriori - finisce per legittimare la scelta di perseguire una vasta politica di accordi elettorali aperta al centro. Non è possibile affrontare in dettaglio le implicazioni politiche di un consenso elettorale così stabile. Qui mi limito a ricordare i riflessi territoriali di questa rigidità: la cintura rossa che comprende quattro regioni mantiene così intatta la sua distintività rispetto alle altre aree del paese. Gli esiti del voto maggioritario nei collegi di questa zona, descritti da D'Alimonte e Bartolini nel saggio che segue, sono del resto eloquenti. Gli unici seggi perduti da Ulivo e Progressisti sono collocati ai margini estremi di questa zona: a Piacenza, Grosseto e San Benedetto del Tronto. Per la Zona rossa si conferma così l'immagine di fortezza inespugnabile certo, ma pur sempre assediata!

In materia di geografia elettorale l'ultima considerazione deve essere dedicata alla Lega. La sua crescita nelle regioni del 
Nord, per quanto tutt'altro che uniforme, costituisce un ulteriore aggravamento della questione territoriale in Italia. Attraverso l'analisi statistica ho cercato di accertare se il ' 96 costituisce una tappa di atvicinamento tra le diverse Italie elettorali oppure se, al contrario, ha marcato un approfondimento delle fratture. Grazie soprattutto ai guadagni di An al Nord e di Fi al Sud queste diverse Italie sembrano più vicine oggi di due anni fa.

È bene tuttavia essere consapevoli che su questo punto la statistica non può essere di consolazione. Fino a un decennio fa il problema della geografia elettorale aveva un rilievo prevalentemente scientifico, e costituiva una via per rispondere agli interrogativi sulle basi sociali del consenso ai diversi partiti e per acquisire indicatori sui valori prevalenti nelle diverse aree. Per il resto, poteva scaldare in maniera intermittente solo l'interesse dei candidati, interessati a individuare circoscrizioni sicure per correre meno rischi possibile. Oggi, con la Lega, le fratture territoriali (che in Italia sono sempre state forti) hanno avuto direttamente accesso al sistema dei partiti. L'interrogativo su quante «Italie» esistano è diventato addirittura, dopo il successo riportato dalla Lega il 21 aprile, una issue di primario rilievo nel confronto politico. Oggi, quindi, l'analisi statistica non può più bastare per fare il punto sulla profondità delle fratture politico-elettorali in Italia; oltretutto, la frattura territoriale posta dalla Lega è così netta che è quasi intrattabile con tecniche statistiche. Con la Lega il problema assume esplicitamente uno spessore politico. L'analisi dei dati, se può essere di aiuto, certo non può surrogare la riflessione teorica e la valutazione politica sulla gravità delle fratture territoriali nell'Italia uscita dalle elezioni del '96. Rifugiarsi nell'analisi statistica significherebbe fare solo dell'accademia, la più arida.

\section{Riferimenti bibliografici}

Agnew, J. (1995), The Rethoric of Regionalism: The Northern League in Italian Politics, 1983-1994, in «Transactions of the Institute of British Geographers», XX, pp. 156-172.

- (1996), Mapping Politics: How Context Counts in Electoral Geography, in «Political Geography», XV, pp. 129-146.

Asher, W. e S. Tarrow (1975), The Stability of Communist Electorates: Evidence from Longitudinal Analysis of French and Italian Aggregate Data, in «American Journal of Political Science», X, pp. 475499. 
Bagnasco, A. (1995), L'Italia in tempo di cambiamento politico, Bologna, Il Mulino.

- (1996), Geografia elettorale e basi sociali della politica, in «Rassegna Italiana di Sociologia», XXXVII, pp. 279-286.

Barbagli, M. e altri (1979), Fluidità elettorale e classi sociali in Italia, Bologna, Il Mulino.

Bartolini, S. (1986), La volatilità elettorale, in «Rivista Italiana di Scienza Politica», XVI, pp. 363-400.

Bartolini, S. e R. D’Alimonte (a cura di) (1995), Maggioritario ma non troppo, Bologna, Il Mulino.

Caciagli, M. e A. Spreafico (a cura di) (1975), Un sistema politico alla prova. Studi sulle elezioni politiche del 1972, Bologna, Il Mulino.

Cartocci, R. (1990), Elettori in Italia. Riflessioni sulle vicende elettorali degli anni ottanta, Bologna, Il Mulino.

- (1996), L'Italia unita dal populismo, in «Rassegna Italiana di Sociologia», XXXVII, pp. 287-295.

Converse, P.E. (1969), Survey Research and the Decoding of Patterns in Ecological Data, in M. Dogan e S. Rokkan (a cura di), Quantitative Ecological Analysis in the Social Sciences, Cambridge, Mit Press, pp. 459-485.

Corbetta, P. e A. Parisi (1994), Il referendum sulla legge elettorale per il Senato, in Mershon e Pasquino, pp. 141-160.

- (1997a), Introduzione, in Corbetta e Parisi (1997b).

- (1997b), A domanda risponde. Il cambiamento del voto degli italiani nel 1994 e nel 1996, Bologna, Il Mulino, in corso di pubblicazione.

Corbetta, P., A. Parisi e H. Schadee (1988), Elezioni in Italia. Struttura e tipologia delle consultazioni politiche, Bologna, Il Mulino.

D'Alimonte, R. (1994), L'uninominale incompiuto, in «il Mulino», $\mathrm{n}$. 351, pp. 55-61.

- (1995), La transizione italiana: il voto regionale del 23 aprile, in «Rivista Italiana di Scienza Politica», XXV, pp. 515-558.

D'Alimonte, R. e S. Bartolini (1995), Il sistema partitico: una transizione difficile, in Bartolini e D'Alimonte, pp. 427-466.

D'Alimonte, R. e A. Chiaramonte (1995), Il nuovo sistema elettorale italiano: le opportunità e le scelte, in Bartolini e D'Alimonte, pp. 37-81.

Diamanti, I. (1995), La Lega, Roma, Donzelli, $2^{\mathrm{a}}$ ed.

Diamanti, I. e R. Mannheimer (a cura di) (1994), Milano a Roma. Guida all'Italia elettorale del 1994, Roma, Donzelli.

Di Virgilio, A. (1996), Le elezioni regionali e amministrative: bipolarizzazione con riserva, in M. Caciagli e D. Kertzer (a cura di), Politica in Italia. Edizione 1996, Bologna, Il Mulino, pp. 57-86.

Dodd, L.C. (1974), Party Coalitions in Multiparty Parliaments: GameTheoretic Analysis, in «Americal Political Science Review», LXVIII, pp. 1043-1117. 
Fabbrini, S. (1996), Luci e ombre della transizione, in «il Mulino», n. 265, pp. 459-467.

Follini, M. (1996), Perché il Polo ba perso le elezioni, in «il Mulino», n. 265 , pp. $468-477$.

Gasperoni, G. (1995), Dimensioni della fluidità elettorale, in Parisi e Schadee, pp. 321-355.

Hartwig, F. (1979), Exploratory Data Analysis, Beverly Hills, Sage.

Mannheimer, R. e G. Sani (1987), Il mercato elettorale, Bologna, Il Mulino.

- (1994), La rivoluzione elettorale. L'Italia fra la prima e la seconda Repubblica, Milano, Anabasi.

Masters, R.D. (1960), Une méthode pour mesurer la mobilité des attitudes politiques, in «Revue Française de Science Politique», X, pp. 658-672.

Mershon, C. e G. Pasquino (a cura di) (1994), Politica in Italia. Edizione 1994, Bologna, Il Mulino.

Natale, P. (1994), La nuova mappa geopolitica, in Diamanti e Mannheimer, pp. 85-98.

Pappalardo, A. (1995) La nuova legge elettorale in parlamento: chi, come e perché, in Bartolini e D'Alimonte, pp. 13-36.

Parisi, A. (1980), Mobilità non significa movimento, in A. Parisi (a cura di), Mobilità senza movimento. Le elezioni del 3 giugno 1979, Bologna, Il Mulino, pp. 11-40.

- (1995), Il difficile mestiere dell'elettore: proposte, alternative, scelte, relazione al Primo Bologna Seminar Italia-Usa, Bologna, $1^{\circ}$ aprile 1995.

Parisi, A. e H. Schadee (a cura di) (1995), Sulla soglia del cambiamento. Elettori e partiti alla fine della prima Repubblica, Bologna, Il Mulino.

Pasquino, G. (1995), Le regole e gli attori, in G. Pasquino (a cura di), L'alternanza inattesa. Le elezioni del 27 marzo 1994 e le sue conseguenze, Soveria Mannelli, Rubbettino, pp. 5-19.

Pedersen, M.N. (1978), La misurazione del mutamento nei sistemi politici: una critica, in «Rivista Italiana di Scienza Politica», VIII, pp. 243-261.

- (1979), The Dynamics of European Party Systems: Changing Patterns of Electoral Volatility, in «European Journal of Political Research», VII, pp. 1-27.

- (1983), Changing Patterns of Electoral Volatility in European Party Systems: 1948-77. Explorations in Explanation, in H. Daalder e P. Mair (a cura di), Western European Party Systems: Continuity and Change, Beverly Hills, Sage.

Przeworski, A. (1975), Institutionalization of Voting Patterns or is Mobilization the Source of Decay?, in «Americal Political Science Review», LXIX, pp. 49-67. 
Rae, D.W. (1971), The Political Consequences of Electoral Laws, New Haven, Yale University Press.

Ricolfi, L. (1995), Il voto proporzionale: il nuovo spazio politico italiano, in Bartolini e D'Alimonte, pp. 273-315.

- (1996), Quali Italie? Vecchie e nuove fratture territoriali, in «Rassegna Italiana di Sociologia», XXXVII, pp. 267-278.

Ruffilli, R. (a cura di) (1987), Materiali per la riforma elettorale, Bologna, Il Mulino.

Sani, G. (1992), 1992: la destrutturazione del mercato elettorale, in «Rivista Italiana di Scienza Politica», XXII, pp. 539-565.

- (1993), Le Italie del 5 aprile, in «Polis», VII, pp. 207-227.

- (1996), I verdetti del 21 aprile, in «il Mulino», n. 265, pp. 451458.

Sartori, G. (1976), Parties and Party Systems: A Framework for Analysis, New York, Cambridge University Press.

- (1982), Teoria dei partiti e caso italiano, Milano, SugarCo.

Spreafico, A. (1968), Le previsioni elettorali, in M. Dogan e O.M. Petracca (a cura di), Partiti politici e strutture sociali in Italia, Milano, Comunità, pp. 121-163.

Tukey, J.W. (1978), Exploratory Data Analysis, Addison-Wesley, Reading.

Vassallo, S. (1997), Struttura della competizione e risultato elettorale, in Corbetta e Parisi (1997b). 